Article

\title{
Synthetic, Structural, and Anticancer Activity Evaluation Studies on Novel Pyrazolylnucleosides
}

\author{
Yogesh Yadav 1,2,3, Deepti Sharma 1,4, Kumar Kaushik ${ }^{5}$, Vineet Kumar 1,6,7, Amitabh Jha ${ }^{2}$, \\ Ashok K. Prasad ${ }^{1}$, Christophe Len ${ }^{8, *} \mathbb{C}$, Sanjay V. Malhotra ${ }^{6,7}$, Jesper Wengel ${ }^{9} \mathbb{D}$ and \\ Virinder S. Parmar 1,5,9,* \\ 1 Bioorganic Laboratory, Department of Chemistry, University of Delhi, Delhi 110 007, India; \\ yogeshyadav77@gmail.com (Y.Y.); sharma81deepti@gmail.com (D.S.); iamvineet@gmail.com (V.K.); \\ ashokenzyme@gmail.com (A.K.P.) \\ 2 Medicinal Chemistry Laboratory, Department of Chemistry, Acadia University, \\ Wolfville, NS B4P 2R6, Canada; amitabh.jha@acadiau.ca \\ 3 SUN Pharmaceuticals R\&D, Gurgaon, Sarhaul, Sector-18, Haryana-122 015, India \\ 4 Sri Venkateswara College, Benito Juarez Road, Dhaula Kuan, University of Delhi, Delhi 110 021, India \\ 5 Department of Chemistry and Environmental Science, Medgar Evers College, The City University of New \\ York, 1638 Bedford Avenue, Brooklyn, NY 11225, USA; kumar.kaushik.drdo@gmail.com \\ 6 Laboratory of Synthetic Chemistry, Leidos Biomedical Research Inc., Frederick National Laboratory for \\ Cancer Research, Frederick, MD 2170, USA; smalhotra@stanford.edu \\ 7 Department of Radiation Oncology, Stanford University, 1050A Arastradero Road, A252, \\ Palo Alto, CA 94304, USA \\ 8 Chimie ParisTech, PSL University, CNRS Institute of Chemistry for Life and Health Sciences-i-CLeHS, \\ 11 rue Pierre et Marie Curie, F-75005 Paris, France \\ 9 Nucleic Acid Center, Department of Physics, Chemistry and Pharmacy, University of Southern Denmark, \\ Campusvej 55, DK-5230 Odense, Denmark; jwe@sdu.dk \\ * Correspondence: christophe.len@chimieparistech.psl.eu (C.L.); vparmar@mec.cuny.edu (V.S.P.); \\ Tel.: +33-(0)638-500-976 (C.L.); Fax: +33-(0)344-971-591 (C.L.)
}

Received: 13 September 2019; Accepted: 28 October 2019; Published: 30 October 2019

check for updates

\begin{abstract}
The synthesis of novel pyrazolylnucleosides 3a-e, 4a-e, 5a-e, and 6a-e are described. The structures of the regioisomers were elucidated by using extensive NMR studies. The pyrazolylnucleosides $5 \mathbf{a}-\mathbf{e}$ and $\mathbf{6 a}-\mathbf{e}$ were screened for anticancer activities on sixty human tumor cell lines. The compound 6 e showed good activity against 39 cancer cell lines. In particular, it showed significant inhibition against the lung cancer cell line Hop-92 $\left(\mathrm{GI}_{50} 9.3 \mu \mathrm{M}\right)$ and breast cancer cell line HS $578 \mathrm{~T}\left(\mathrm{GI}_{50} 3.0 \mu \mathrm{M}\right)$.
\end{abstract}

Keywords: pyrazolylnucleosides; modified nucleosides; NOESY study; anticancer; NCI-60

\section{Introduction}

The chemistry of nucleosides has been extensively studied and several analogs have been found to exhibit potential as fungicidal, antitumor, and antiviral agents [1-7]. Modifications in both the heterocyclic bases and the sugar moieties have led to active and safer nucleoside analogues that have found applications as agents effective against human immunodeficiency virus (HIV), the causative agent of acquired immune deficiency syndrome (AIDS), and also against viral infections caused by the herpes simplex virus (HSV types 1 and 2), varicella zoster virus (VZV), hepatitis $\mathrm{C}$ virus (HCV), human cytomegalovirus (HCMV), and Epstein-Barr virus (EBV) [8,9]. Nucleoside and nucleotide modifications resulted in an increased interest in the regio- and stereoselective synthesis of nucleosides $[10,11]$. Moreover, modified nucleosides and nucleotides with a restricted 
conformation have been used to reach a particular conformation of a rotamer to study the affinity of a biomacromolecule for its natural ligand as well as the molecular recognition in an oligonucleotide chain (RNA/DNA) [12-15]. Similar studies of anti-sense and anti-gene oligonucleotides (ONs) as potential and selective inhibitors of gene expression [16-19] and their use as anti-tumor or anti-viral agents [20-23] have also influenced the developments in the field of nucleic acid-based drugs. Among the nucleoside analogues with significant biological activities, dideoxynucleoside-based compounds such as $2^{\prime}, 3^{\prime}$-dideoxycytidine (ddC) [24], 2' , 3' -dideoxyinosine (ddI) [24], and 3' -azidothymidine (AZT) [25] are effective therapeutic agents for the treatment of AIDS, while ribavirin (virazole) $[26,27]$ is an antiviral drug (Figure 1). Similarly, other dideoxynucleosides such as d4T ( $2^{\prime}, 3^{\prime}$-didehydro- $3^{\prime}$-deoxythymidine, stavudine) [28,29] and AZddU ( $3^{\prime}$-azido-2', $3^{\prime}$-dideoxyuridine) [30] have gone through clinical studies. Different nucleosides isolated from nature such as oxazinomycin [31], pyrazofurin [32,33], showdomycin [34,35], formycin A, and formycin B [36,37] have shown antibiotic properties and have also been found to exhibit anticancer and/or antiviral activities (Figure 1). These examples and new developments in the chemistry and biology of these compounds and their analogs [38-42] have motivated us to work in this area and have led us to investigate the interesting chemical and biological properties of novel nucleoside-based compounds.<smiles>Cc1cn(C2CC(N)C(CO)O2)c(=O)[nH]c1=O</smiles>

AZT Showdomycin<smiles>NC(=O)c1ncn(C2OC(CO)C(O)C2O)n1</smiles>

Ribavirin<smiles>NC(=O)c1[nH]nc(C2OC(CO)C(O)C2O)c1O</smiles>

Pyrazofurin<smiles>Nc1ncnc2c(C3OC(CO)C(O)C3O)n[nH]c12</smiles>

Formycin A Formycin B

Figure 1. Selected nucleoside analogues with antiviral and/or antitumoral activities: 3 '-azidothymidine (AZT or ZDV), showdomycin, ribavirin, pyrazofurin, formycin A and formycin B.

Despite the developments in nucleoside chemistry, the clinical use of nucleosides has some drawbacks due to their side-effects and primary or acquired drug resistance [43]. Therefore, the search for the design of new and effective nucleoside-based analogues continues to motivate studies in this field. Our efforts toward this goal have led to the synthesis of twenty novel pyrazolylnucleosides with new structures and potent antiviral and antitumoral behaviors. In this regard, two regioisomers for each pyrazole derivative have been produced and characterized using spectroscopic techniques such as ${ }^{1} \mathrm{H}$ NMR, ${ }^{13} \mathrm{C}$ NMR, NOESY, HMBC, IR, and mass spectroscopy. All compounds were evaluated against the National Cancer Institute (NCI)'s panel of 60 human tumor cell lines for their anticancer activities, and for their antiviral activities against representative viruses.

\section{Results and Discussion}

\subsection{Chemical Synthesis of the Nucleoside Analogues 5 and 6}

In our approach, the synthesis of the target compounds $\mathbf{5 a - e}$ and $\mathbf{6 a - e}$ was achieved in two steps starting from the already reported 3-cyanomethyl-5-aryl-1H-pyrazoles 1a-e [44] and the well-known 2-deoxy-3,5-di-O- $p$-toluoyl- $\alpha$-D-ribofuranosyl chloride (2) [45] (Scheme 1). Treatment of pyrazoles 1a-e with sodium hydride in acetonitrile and the subsequent addition of chlorosugar $\mathbf{2}$ gave two regioisomers of the modified pyrazolyl nucleosides 3a-e (by coupling 2 with the $N-1$ nitrogen of the pyrazole derivatives) and $4 \mathbf{a}-\mathbf{e}$ (by coupling chlorosugar 2 with the N-2 nitrogen of the pyrazole derivatives) in 58-65\% yields. The products $3 \mathbf{a}-\mathbf{e}$ and $4 \mathbf{a}-\mathbf{e}$ were de-toluoylated by using sodium 
methoxide in methanol, resulting in the formation of the corresponding modified nucleosides 5a-e and $\mathbf{6 a - e}$ in $70-80 \%$ yields, respectively.

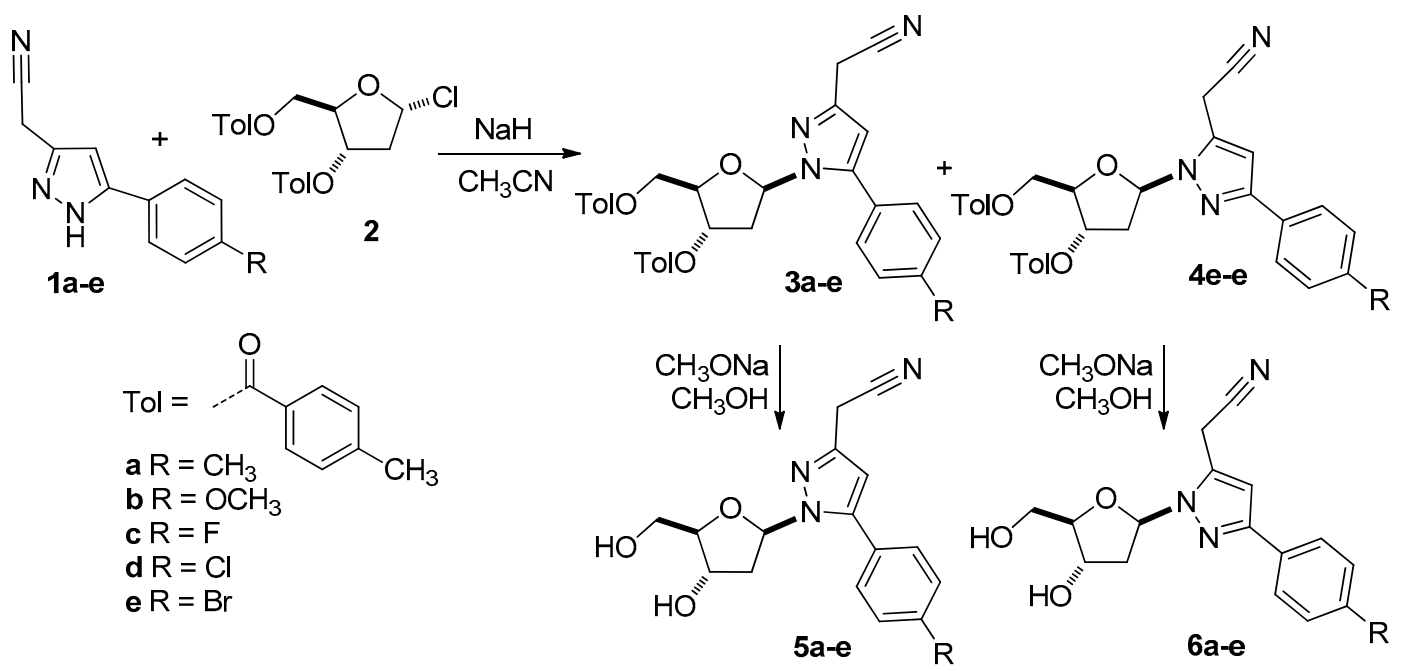

Scheme 1. Synthesis of pyrazolylnucleoside analogues 5a-e and $\mathbf{6 a - e . ~}$

Pyrazoles with no substitution on either of the two $\mathrm{N}$ ring atoms can be alkylated to produce two regioisomers under strongly basic conditions. The anion generated produces the two resonance forms that react with chlorosugar $\mathbf{2}$ in the glycosidation step, leading to mixtures of two regioisomeric pyrazolyl nucleosides (i.e., 3a-e and $\mathbf{4 a - e}$ ) (Scheme 2).

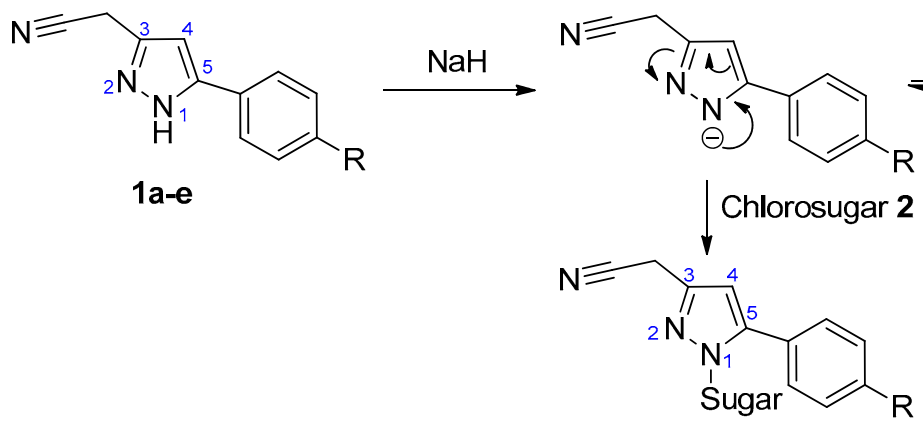

3a-e

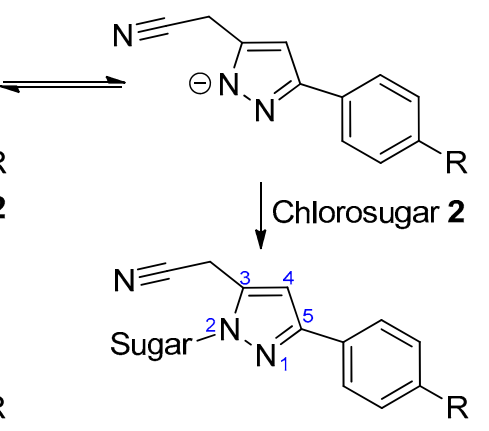

4a-e

Scheme 2. Mechanistic explanation for the regioisomeric alkylation of pyrazole derivatives 1a-e. The IUPAC numbering scheme of $\mathbf{3 a - e}$ is retained in $\mathbf{4 a - e}$ (and in $\mathbf{5 a - e}$ and $\mathbf{6 a}-\mathbf{e}$ ) for easy comparison.

\subsection{Structural Identification of the Isomeric Pyrazolyl Nucleoside Analogues 3-6}

The structural identification of the isomeric disubstituted pyrazolyl nucleosides, based on their ${ }^{1} \mathrm{H}$ NMR spectral data (See Supplementary Materials), has been reported in the literature [46-48]. It has been observed that the anomeric protons in the isomeric pyrazolyl nucleosides exhibit different proton chemical shifts. The anomeric proton adjacent to $N-1$ of the pyrazole compounds $3 \mathbf{a}-\mathbf{e}$ and $\mathbf{5 a}-\mathbf{e}$ would appear downfield when compared to the anomeric proton adjacent to $\mathrm{N}-2$ of the pyrazole compounds 4a-e and 6a-e [46-48]. In addition to using ${ }^{1} \mathrm{H}$ NMR chemical shifts, extensive NOE and 2D NMR experiments have been employed to confirm the structures of the isomeric pyrazolyl nucleosides. Using ${ }^{1} \mathrm{H}$ and ${ }^{13} \mathrm{C}$ NMR studies, we confirmed the positional assignments of the isomeric pyrazolyl nucleosides synthesized in the present work (Tables 1-3). The anomeric proton of the analogues 5a-e generally appeared at $0.14-0.19 \mathrm{ppm}$ upfield when compared to the corresponding proton in the ${ }^{1} \mathrm{H}$ NMR spectra of its corresponding 6 series isomers. This is in agreement with observations for other 1,5- and 1,3-disubstituted pyrazole nucleosides [46-48]. We found that the effect was less pronounced 
in the $\mathbf{3}$ series of nucleosides as compared to the $\mathbf{4}$ series nucleosides where this difference was in the range of $0.04-0.14 \mathrm{ppm}$. The ${ }^{13} \mathrm{C}$ chemical shift of the $-\mathrm{CH}_{2} \mathrm{CN}$ bearing pyrazole carbon atom can help distinguish between positional isomers. In the 3 and 5 series of nucleosides, this carbon signal was 4-11 ppm downfield relative to the corresponding signal in the 4 and $\mathbf{6}$ series nucleosides. In most cases, the aryl bearing pyrazole carbon in the 3 and 5 series was $4-7$ ppm upfield relative to the corresponding carbon in the $\mathbf{4}$ and $\mathbf{6}$ series. However, in the case of $\mathbf{3 d}$ versus $\mathbf{4 d}$, the difference was small and reversed, making the aryl bearing pyrazole carbon less reliable for use in positional assignments.

Table 1. Chemical shift values of the anomeric protons in the ${ }^{1} \mathrm{H}$ NMR spectra and chemical shift values of the Ar- and $\mathrm{CH}_{2} \mathrm{CN}$ bearing carbons of the pyrazole ring in the ${ }^{13} \mathrm{C}$ NMR spectra of the isomeric pyrazolyl nucleosides $3 \mathbf{a}-\mathbf{e}, \mathbf{4 a}-\mathbf{e}, \mathbf{5 a}-\mathbf{e}$, and $\mathbf{6 a}-\mathbf{e}$ in $\mathrm{CDCl}_{3}$ on a Bruker Avance 300 spectrometer.

\begin{tabular}{|c|c|c|c|c|c|c|}
\hline \multirow[t]{2}{*}{ Compound } & \multicolumn{2}{|c|}{$\begin{array}{c}\text { C-1' H Shift in the } \\
{ }^{1} \mathrm{H} \text { NMR Spectrum (ppm) }\end{array}$} & \multicolumn{2}{|c|}{$\begin{array}{l}\text { Ar- Bearing C Shifts in the } \\
{ }^{13} \text { C NMR Spectrum (ppm) }\end{array}$} & \multicolumn{2}{|c|}{$\begin{array}{l}-\mathrm{CH}_{2} \mathrm{CN} \text { Bearing C Shifts in } \\
\text { the }{ }^{13} \mathrm{C} \text { NMR Spectrum (ppm) }\end{array}$} \\
\hline & Series 3 & Series 5 & Series 3 & Series 5 & Series 3 & Series 5 \\
\hline $\mathbf{a}$ & 6.13 & 5.99 & 144.6 & 145.9 & 141.7 & 142.0 \\
\hline b & 6.10 & 6.02 & 146.2 & 145.8 & 144.0 & 141.8 \\
\hline c & 6.05 & 5.97 & 145.4 & 144.9 & 141.9 & 141.9 \\
\hline d & 6.05 & 6.00 & 145.2 & 144.7 & 143.7 & 141.9 \\
\hline \multirow[t]{2}{*}{ e } & 6.05 & 6.00 & 145.6 & 143.9 & 142.3 & 141.1 \\
\hline & Series 4 & Series 6 & Series 4 & Series 6 & Series 4 & Series 6 \\
\hline a & 6.17 & 6.13 & 151.0 & 150.2 & 137.9 & 137.7 \\
\hline b & 6.16 & 6.19 & 150.8 & 150.7 & 133.0 & 133.7 \\
\hline c & 6.17 & 6.16 & 152.5 & 149.8 & 135.8 & 135.8 \\
\hline d & 6.19 & 6.17 & 144.1 & 149.6 & 134.3 & 134.6 \\
\hline e & 6.17 & 6.18 & 149.8 & 149.2 & 133.5 & 134.9 \\
\hline
\end{tabular}

Table 2. Chemical shift and coupling constants from the ${ }^{1} \mathrm{H}-\mathrm{NMR}$ of compounds $\mathbf{3 d}, \mathbf{4 d}, \mathbf{5 d}$, and $\mathbf{6} \mathbf{d}$ in acetone- $d_{6} \cdot{ }^{\text {a }}$ Please refer to structures in Table 4 for skeleton numbering.

\begin{tabular}{|c|c|c|c|c|c|c|c|c|}
\hline \multirow{2}{*}{ Proton } & \multicolumn{4}{|c|}{ Chemical Shifts $(\delta)$} & \multicolumn{4}{|c|}{ Coupling Constants (Hz) } \\
\hline & $3 d$ & $4 d$ & $5 d$ & $6 d$ & $3 d$ & $4 d$ & $5 d$ & $6 d$ \\
\hline $\mathrm{H} 1^{\prime}$ & 6.29 & 6.48 & 6.09 & 6.29 & dd $5.7,6.5$ & dd $6.5,5.2$ & $t^{*} 6.4$ & $t^{*} 6.2$ \\
\hline $\mathrm{H} 2^{\prime} \alpha$ & 2.72 & 2.79 & 2.31 & 2.42 & $\begin{array}{c}\text { ddd } \\
14.0,6.7,4.1\end{array}$ & $\begin{array}{c}\text { ddd } \\
14.0,4.5,6.6\end{array}$ & $\begin{array}{c}\text { ddd } \\
13.5,3.7,6.8\end{array}$ & $\begin{array}{c}\text { ddd } \\
13.5,3.7,6.7\end{array}$ \\
\hline $\mathrm{H} 2^{\prime} \beta$ & 3.54 & 3.63 & 3.01 & 3.00 & $\begin{array}{c}\text { ddd } \\
14.0,6.8,5.6\end{array}$ & $\begin{array}{c}\text { ddd } \\
14.0,5.2,6.7\end{array}$ & $\begin{array}{c}\mathrm{dt}^{*} \\
13.5,6.0\end{array}$ & $\begin{array}{c}\mathrm{dt}^{*} \\
13.5,5.9\end{array}$ \\
\hline $\mathrm{H}^{\prime}$ & 5.93 & 5.97 & 4.66 & 4.70 & $\mathrm{~m}$ & $\mathrm{~m}$ & $\mathrm{~m}$ & $\mathrm{~m}$ \\
\hline $\mathrm{H} 4^{\prime}$ & 4.62 & 4.65 & 4.02 & 4.07 & $\mathrm{~m}$ & $\mathrm{~m}$ & $\mathrm{~m}$ & $\mathrm{~m}$ \\
\hline $\mathrm{H} 5^{\prime} \alpha$ & 4.54 & 4.45 & 3.61 & 3.59 & $\mathrm{~m}$ & dd $13.2,6.4$ & ddd $11.8,4.8,7.7$ & ddd $11.9,4.1,8.1$ \\
\hline $\mathrm{H} 5^{\prime} \beta$ & 4.63 & 4.63 & 3.70 & 3.71 & $\mathrm{~m}$ & $\mathrm{~m}$ & $\mathrm{dt}^{*} 11.9,4.7$ & $\mathrm{dt}^{*} 11.9,4.1$ \\
\hline $\mathrm{H} 4$ & 6.50 & 6.82 & 6.46 & 6.83 & brs, $1 \mathrm{H}$ & $t^{*} 0.8$ & $t^{*} 0.5$ & t 0.8 \\
\hline H6 & 3.90 & 4.29 & 3.96 & 4.31 & brs, $2 \mathrm{H}$ & $19.0,0.8$ & d 0.5 & $18.9,0.8$ \\
\hline $\mathrm{H} 2^{\prime \prime}$ & 7.67 & 7.87 & 7.60 & 7.87 & & & & \\
\hline $\mathrm{H}^{\prime \prime}$ & & & 7.57 & 7.46 & & & & \\
\hline
\end{tabular}

${ }^{\text {a }}$ The chemical shift values presented in Table 2 were all measured in acetone- $d_{6}$ (on an Bruker Avance 400 instrument) and may therefore differ from those in Table 1, which were measured in $\mathrm{CDCl}_{3}$ (on Bruker Avance 300 instrument). ${ }^{*}$ Due to the spatial interactions between different protons as explicitly shown in Table 4, these protons exhibited multiplicities as shown here when the spectra were recorded in acetone- $d_{6}$ on a Bruker Avance 400 instrument as against those recorded in $\mathrm{CDCl}_{3}, \mathrm{CD}_{3} \mathrm{CN}$ or DMSO- $d_{6}$ as given in the Experimental section for the corresponding protons where they appeared as broad singlets or ill resolved multiplets when their spectra were recorded on a Bruker Avance 300 instrument. 
Table 3. Chemical shift values from the ${ }^{13} \mathrm{C}-\mathrm{NMR}$ of compounds $3 \mathrm{~d}, \mathbf{4 d}, \mathbf{5 d}$, and $\mathbf{6 d}$ in acetone- $d_{6} \cdot{ }^{\mathrm{a}}$ Please refer to the structures in Table 4 for skeleton numbering.

\begin{tabular}{|c|c|c|c|}
\hline \multicolumn{4}{|c|}{ Chemical Shifts ( $\delta)$ (Carbon Position) } \\
\hline $3 d$ & $5 d$ & $4 d$ & $6 d$ \\
\hline $87.6\left(\mathrm{C1}^{\prime}\right)$ & $87.4\left(\mathrm{C1}^{\prime}\right)$ & $87.6\left(\mathrm{C1}^{\prime}\right)$ & $88.0\left(\mathrm{C}^{\prime}\right)$ \\
\hline $37.1\left(\mathrm{C}^{\prime}\right)$ & $40.5\left(\mathrm{C}^{\prime}\right)$ & $37.0\left(\mathrm{C}^{\prime}\right)$ & $41.1\left(\mathrm{C}^{\prime}\right)$ \\
\hline $76.6\left(\mathrm{C}^{\prime}\right)$ & $73.2\left(\mathrm{C}^{\prime}\right)$ & $76.1\left(\mathrm{C}^{\prime}\right)$ & $73.0\left(\mathrm{C}^{\prime}\right)$ \\
\hline $83.5\left(\mathrm{C} 4^{\prime}\right)$ & $89.8\left(\mathrm{C}^{\prime}\right)$ & $83.7\left(\mathrm{C}^{\prime}\right)$ & $90.0\left(\mathrm{C} 4^{\prime}\right)$ \\
\hline $65.1\left(\mathrm{C}^{\prime}\right)$ & $64.2\left(\mathrm{C}^{\prime}\right)$ & $64.7\left(\mathrm{C}^{\prime}\right)$ & $63.9\left(\mathrm{C}^{\prime}\right)$ \\
\hline $143.8(\mathrm{C} 3)$ & $143.6(\mathrm{C} 3)$ & $136.3(\mathrm{C} 3)$ & $136.1(\mathrm{C} 3)$ \\
\hline $107.2(\mathrm{C} 4)$ & $106.7(\mathrm{C} 4)$ & $105.4(\mathrm{C} 4)$ & $104.9(\mathrm{C} 4)$ \\
\hline $146.0(\mathrm{C} 5)$ & $145.8(\mathrm{C} 5)$ & $150.6(\mathrm{C} 5)$ & $150.5(\mathrm{C} 5)$ \\
\hline $17.6(\mathrm{C} 6)$ & $17.6(\mathrm{C} 6)$ & $15.3(\mathrm{C} 6)$ & $15.2(\mathrm{C} 6)$ \\
\hline $118.1(\mathrm{CN})$ & $118.0(\mathrm{CN})$ & $117.0(\mathrm{CN})$ & $117.1(\mathrm{CN})$ \\
\hline $166.4\left(\mathrm{C6}^{\prime \prime \prime}\right)$ & & $166.5\left(\mathrm{C}^{\prime \prime \prime}\right)$ & \\
\hline $166.5\left(\mathrm{C}^{\prime \prime \prime \prime \prime}\right)$ & & $166.5\left(\mathrm{C}^{\prime \prime \prime \prime \prime}\right)$ & \\
\hline * & $129.3\left(C 1^{\prime \prime}\right)$ & $\#$ & $134.2\left(\mathrm{C} 1^{\prime \prime}\right)$ \\
\hline * & $131.6\left(\mathrm{C}^{\prime \prime}\right)$ & $\#$ & $127.9\left(\mathrm{C} 2^{\prime \prime}\right)$ \\
\hline * & $129.9\left(\mathrm{C}^{\prime \prime}\right)$ & $\#$ & $129.7\left(\mathrm{C}^{\prime \prime}\right)$ \\
\hline * & $135.6\left(\mathrm{C}^{\prime \prime}\right)$ & $\#$ & $132.6\left(\mathrm{C}^{\prime \prime}\right)$ \\
\hline
\end{tabular}

a The chemical shift values presented in Table 3 were all measured in acetone- $\mathrm{d}_{6}$ (on an Bruker Avance 400 instrument) and may therefore differ from those in Table 1, which were measured in $\mathrm{CDCl}_{3}$ (on Bruker Avance 300 instrument). * Benzene ring Carbons: Quaternary C: 145.0, 144.7, 135.6, 129.3, 128.3, 128.1; CHs: 131.6, 130.5, 130.5, 130.0, 130.0, 129.9. \# Benzene ring Carbons: Quaternary C: 145.0, 144.5, 134.1, 132.7, 128.1; CHs: 130.5, 130.4, 130.1, 129.9, 129.5.

In order to confirm the above positional assignments, we performed 2D NMR experiments (NOESY and HMBC) on the two pairs of compounds (i.e., one pair consisting of the ditoluoyl protected nucleosides $\mathbf{3} \mathbf{d}$ and $\mathbf{4} \mathbf{d}$ and another pair consisting of the deprotected nucleosides $\mathbf{5} \mathbf{d}$ and $\mathbf{6} \mathbf{d}$ ) (Table 4 ). The results of the HMBC experiments rely on the fact that the anomeric proton may see the aryl bearing pyrazole carbon in $\mathbf{3 d}$ and $\mathbf{5 d}$, but not in $\mathbf{4 d}$ and $\mathbf{6 d}$, while the NOESY results would explain the spatial proximity of the anomeric proton to either the protons in the $-\mathrm{CH}_{2} \mathrm{CN}$ group or to the ortho protons of the aryl group. Our results verified the positional properties at the pyrazole ring in the four compounds and are summarized in Table 4.

The isomers of the $\mathbf{3}$ series nucleosides generally had higher $\mathrm{R}_{\mathrm{f}}$ values on the TLC than the corresponding isomer of the 4 series of nucleosides. The same was observed for the isomers of the 5 series relative to those in the 6 series of nucleosides. 
Table 4. Results from the NOESY and HMBC spectra of the pairs $\mathbf{3 d}-\mathbf{4 d}$ and $\mathbf{5 d}-\mathbf{6} \mathbf{d}$ *.
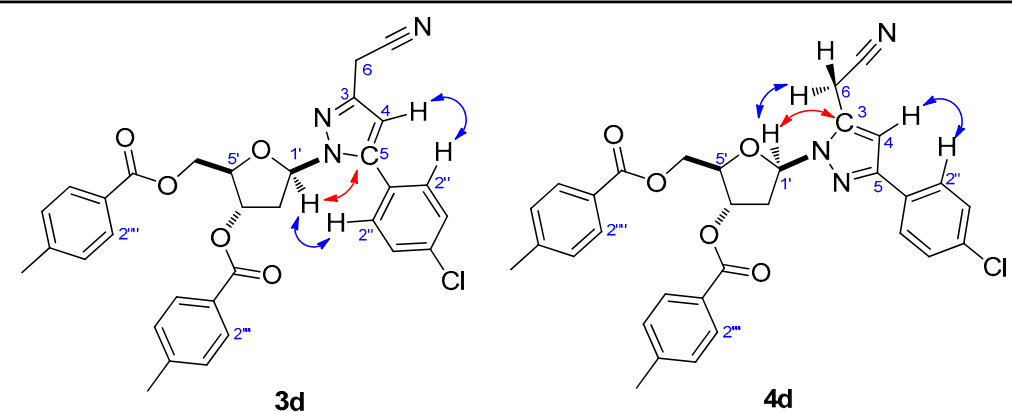

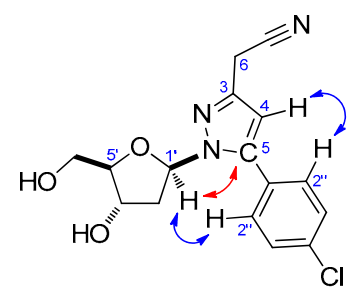

5d

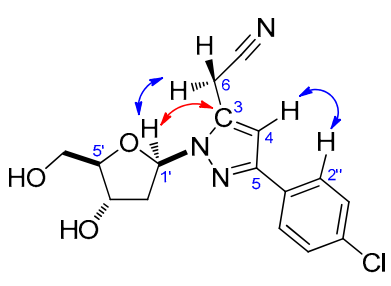

6d

Blue and red arrows indicate the NOESY and HMBC cross peaks, respectively.

\begin{tabular}{ccccc}
\hline & $\mathbf{3 d}$ & $\mathbf{4 d}$ & $\mathbf{5 d}$ & $\mathbf{6 d}$ \\
\hline NOESY H1'-H6 & $\mathrm{o}$ & $\underline{\mathrm{x}}$ & $\mathrm{o}$ & $\underline{\mathrm{x}}$ \\
\hline NOESY H1' ${ }^{\prime}-\mathrm{H} 2^{\prime \prime}$ & $\underline{x}$ & $\mathrm{o}$ & $\underline{x}$ & $\mathrm{o}$ \\
\hline NOESY H4-H2 ${ }^{\prime \prime}$ & $\mathrm{x}$ & $\mathrm{x}$ & $\mathrm{x}$ & $\mathrm{x}$ \\
\hline HMBC H1'-C5 & $\underline{\mathrm{x}}$ & $\mathrm{o}$ & $\underline{\mathrm{x}}$ & $\mathrm{o}$ \\
\hline HMBC H1'-C3 & $\mathrm{o}$ & $\underline{\mathrm{x}}$ & $\mathrm{o}$ & $\underline{\mathrm{x}}$
\end{tabular}

* The presence of a cross peak is indicated by (x). When it contributes to the positional verification, it is underlined (x). See Tables 1-3 for positions of the relevant protons and carbons in the NMR spectra of the compounds $\mathbf{3 d}, \mathbf{4 d}$, $5 \mathrm{~d}$ and $6 \mathrm{~d}$.

\subsection{Anticancer Activity of the Isomeric Pyrazolyl Nucleoside Analogues $5 \boldsymbol{a}-\boldsymbol{e}$ and $\mathbf{6} \boldsymbol{a}-\boldsymbol{e}$}

The anticancer and toxicity of compounds $5 \mathbf{a}-\mathbf{e}$ and $\mathbf{6 a}-\mathbf{e}$ were evaluated by using the National Cancer Institute's 60 human cancer cell lines. Compounds 5a-e, where the sugar is attached to N-1 of the pyrazole ring, were found to be inactive against all the cell lines irrespective of the substituent on the aromatic ring. In the other series, compounds $\mathbf{6 a}, \mathbf{6 b}$, and $\mathbf{6 c}$ with 4-methyl, 4-methoxy, and 4-fluoro substituents, respectively, at the aromatic ring were also inactive. However, compounds $\mathbf{6 d}$ and $\mathbf{6 e}$ with 4-chloro and 4-bromo substituents at the aromatic ring, respectively, showed inhibition against multiple anticancer cell lines. The $\mathrm{GI}_{50}$ (cytostatic parameter) and $\mathrm{LC}_{50}$ (toxicity parameter) values of $\mathbf{6 d}$ and $\mathbf{6 e}$ for the selected cell lines are given in Table 5. Compound $\mathbf{6 d}$ showed inhibition in 19 cell lines, and was most active against the renal cancer cell line UO-31 and breast cancer cell line HS 578T with $\mathrm{GI}_{50}<20 \mu \mathrm{M}$ in both cases. The most active compound was $6 \mathbf{e}$, which showed moderate inhibition in 39 cell lines. It showed significant inhibition against lung cancer cell line Hop-92 with a GI $_{50}$ of $9.3 \mu \mathrm{M}$ and breast cancer cell line HS 578T with a $\mathrm{GI}_{50}$ of $3.0 \mu \mathrm{M}$. Most importantly, both compounds (6d and 6e) did not show any toxicity, even at the highest concentration tested, as indicated by their high $\mathrm{LC}_{50}$ values.

The pyrazolyl nucleosides $\mathbf{5 a}-\mathbf{e}$ and $\mathbf{6 a}-\mathbf{e}$ were also examined for their antiviral activities against a number of viruses such as Simplex virus type 1 (HSV-1) and type 2 (HSV-2), thymidine kinase-deficient (TK-) strains of HSV-1, Vaccinia virus, para-influenza-3 virus, Sindbis virus, Coxsackie virus, Punta toro virus, vesicular stomatitis virus (VSV), Coxsackie virus B4 (CV-B4), respiratory syncytial virus (RSV), feline corona virus, and feline herpes virus. However, none of these compounds showed any significant activity against any of these viruses. 
Table 5. Antitumor activity $\left(\mathrm{GI}_{50} / \mu \mathrm{M}\right){ }^{\mathrm{a}}$ and toxicity $\left(\mathrm{LC}_{50} / \mu \mathrm{M}\right){ }^{\mathrm{b}}$ data of $6 \mathbf{d}$ and 6 e with the anticancer drug dasatinib as a positive control.

\begin{tabular}{|c|c|c|c|c|c|c|}
\hline \multirow{2}{*}{ Panels/Cell Lines } & \multicolumn{2}{|c|}{$6 d$} & \multicolumn{2}{|c|}{$6 e$} & \multicolumn{2}{|c|}{ Dasatinib } \\
\hline & $\mathrm{GI}_{50}$ & $\mathrm{LC}_{50}$ & $\mathrm{GI}_{50}$ & $\mathrm{LC}_{50}$ & $\mathrm{GI}_{50}$ & $\mathrm{LC}_{50}$ \\
\hline \multicolumn{7}{|l|}{ Leukemia } \\
\hline CCRF-CEM & $>67.5$ & $>67.5$ & 46.3 & $>57.5$ & 5.3 & $>100$ \\
\hline K-562 & $>67.5$ & $>67.5$ & 40.0 & $>57.5$ & 0.01 & $>100$ \\
\hline MOLT-4 & $>67.5$ & $>67.5$ & 30.9 & $>57.5$ & 4.1 & $>100$ \\
\hline RPMI-8226 & $>67.5$ & $>67.5$ & 34.0 & $>57.5$ & 4.8 & 99.5 \\
\hline SR & $>67.5$ & $>67.5$ & 25.5 & $>57.5$ & 3.1 & 89.3 \\
\hline \multicolumn{7}{|c|}{ Non-Small Cell Lung Cancer } \\
\hline A549/ATCC & 44.8 & $>67.5$ & 29.3 & $>57.5$ & 0.05 & 75.5 \\
\hline HOP-92 & $>67.5$ & $>67.5$ & 9.3 & $>57.5$ & 0.01 & $>100$ \\
\hline NCI-H322M & 29.8 & $>67.5$ & 30.2 & $>57.5$ & 0.04 & 27.0 \\
\hline NCI-H522 & $>67.5$ & $>67.5$ & 25.7 & $>57.5$ & 0.06 & 55.1 \\
\hline \multicolumn{7}{|l|}{ Colon Cancer } \\
\hline HCT-116 & $>67.5$ & $>67.5$ & 47.7 & $>57.5$ & 11.8 & 69.8 \\
\hline HCТ-15 & $>67.5$ & $>67.5$ & 45.1 & $>57.5$ & 0.6 & 71.1 \\
\hline \multicolumn{7}{|l|}{ CNS Cancer } \\
\hline SF-268 & 33.5 & $>67.5$ & 20.5 & $>57.5$ & 0.07 & 75.5 \\
\hline SF-295 & $>67.5$ & $>67.5$ & 24.8 & $>57.5$ & 1.1 & 46.8 \\
\hline SNB-19 & 24.6 & $>67.5$ & 18.5 & $>57.5$ & 11.9 & 76.4 \\
\hline SNB-75 & 53.5 & $>67.5$ & 10.5 & $>57.5$ & 0.01 & 46.3 \\
\hline U251 & $>67.5$ & $>67.5$ & 36.4 & $>57.5$ & 3.2 & 52.4 \\
\hline \multicolumn{7}{|l|}{ Melanoma } \\
\hline LOX IMVI & $>67.5$ & $>67.5$ & 49.7 & $>57.5$ & 0.01 & 2.8 \\
\hline M14 & $>67.5$ & $>67.5$ & 57.1 & $>57.5$ & 3.1 & 51.5 \\
\hline MDA-MB-435 & $>67.5$ & $>67.5$ & 53.0 & $>57.5$ & 4.1 & 65.5 \\
\hline SK-MEL-2 & $>67.5$ & $>67.5$ & 39.6 & $>57.5$ & 1.1 & 71.8 \\
\hline SK-MEL-5 & 60.9 & $>67.5$ & 11.8 & $>57.5$ & 4.5 & 44.3 \\
\hline UACC-62 & 37.8 & $>67.5$ & 10.5 & $>57.5$ & 2.8 & 47.0 \\
\hline \multicolumn{7}{|l|}{ Ovarian Cancer } \\
\hline IGROV1 & 32.6 & $>67.5$ & 21.5 & $>57.5$ & 0.01 & 80.0 \\
\hline OVCAR-3 & $>67.5$ & $>67.5$ & 39.1 & $>57.5$ & 0.2 & 74.6 \\
\hline OVCAR-5 & $>67.5$ & $>67.5$ & 44.4 & $>57.5$ & 0.02 & 86.3 \\
\hline \multicolumn{7}{|l|}{ Renal Cancer } \\
\hline A498 & 22.6 & $>67.5$ & 18.8 & $>57.5$ & 0.03 & 16.0 \\
\hline $\mathrm{ACHN}$ & $>67.5$ & $>67.5$ & 45.1 & $>57.5$ & 0.01 & 520 \\
\hline CAK-1 & 22.9 & $>67.5$ & 18.8 & $>57.5$ & 0.01 & 5.1 \\
\hline RXF 393 & 38.0 & $>67.5$ & 34.6 & $>57.5$ & 0.03 & 10.4 \\
\hline SN12C & 48.4 & $>67.5$ & 24.3 & $>57.5$ & 0.05 & 44.4 \\
\hline UO-31 & 19.4 & $>67.5$ & 16.6 & $>57.5$ & 0.01 & 82.0 \\
\hline \multicolumn{7}{|l|}{ Prostate Cancer } \\
\hline PC-3 & 37.4 & $>67.5$ & 20.0 & $>57.5$ & 0.2 & 92.5 \\
\hline DU-145 & $>67.5$ & $>67.5$ & 45.1 & $>57.5$ & 0.1 & 4.9 \\
\hline \multicolumn{7}{|l|}{ Breast Cancer } \\
\hline MCF7 & 48.0 & $>67.5$ & 32.8 & $>57.5$ & 12.6 & 71.4 \\
\hline MDA-MB-231ATCC & $>67.5$ & $>67.5$ & 55.8 & $>57.5$ & 0.01 & 38.0 \\
\hline HS 578T & 16.6 & $>67.5$ & 3.0 & $>57.5$ & 0.01 & $>100$ \\
\hline BT-549 & 49.9 & $>67.5$ & 30.0 & $>57.5$ & 5.9 & 49.8 \\
\hline $\mathrm{T}-47 \mathrm{D}$ & 26.6 & $>67.5$ & 19.4 & $>57.5$ & 0.2 & 91.4 \\
\hline MDA-MB-468 & 31.6 & $>67.5$ & 13.8 & $>57.5$ & 0.09 & 8.4 \\
\hline
\end{tabular}

${ }^{\text {a }} \mathrm{GI}_{50}: 50 \%$ Growth inhibition, concentration of drug resulting in a $50 \%$ reduction in net protein increase when compared with the control cells. ${ }^{\mathrm{b}} \mathrm{LC}_{50}$ : Lethal concentration, concentration of drug lethal to $50 \%$ of cells. 


\section{Experimental}

\subsection{General Information}

Reactions were conducted under an atmosphere of nitrogen in anhydrous solvents. Column chromatography was carried out by using silica gel (100-200 mesh). Melting points were determined in a concentrated $\mathrm{H}_{2} \mathrm{SO}_{4}$ acid bath and were uncorrected. Analytical TLCs were performed on pre-coated Merck silica gel $60 \mathrm{~F}_{254}$ plates; the spots were detected either by using UV light or by charring with $4 \%$ alcoholic sulfuric acid. The IR spectra were recorded on a Perkin-Elmer 2000 FT-IR spectrophotometer (Waltham, MA, USA). The optical rotations were measured with a Bellingham-Stanley AD 220 polarimeter and the concentrations expressed as $\mathrm{g} / \mathrm{mL}$. The ${ }^{1} \mathrm{H}-\mathrm{NMR}$ and ${ }^{13} \mathrm{C}-\mathrm{NMR}$ spectra were recorded on a Bruker Avance 300 spectrometer (Billerica, MA, USA) at 300 and $75.5 \mathrm{MHz}$, respectively in $\mathrm{CDCl}_{3}, \mathrm{DMSO}-d_{6}$, or $\mathrm{CD}_{3} \mathrm{CN}$. All 2D measurements were performed in acetone- $d_{6}$ on a Bruker Avance 400 spectrometer (Billerica, MA, USA). Chemical shifts are relative to internal TMS. Assignments were based on COSY, NOESY, HMBC (by using Bruker's microprogram inv4gslplrnd, which includes the low-pass J-filter to suppress one-bond correlations), HSQC, and JRES spectra. The chemical shift values were reported as $\delta$ ppm relative to TMS used as the internal standard and the coupling constants $(J)$ were measured in Hz. The ESI-HRMS spectra of all compounds were recorded on a JEOL JMS-AX505W high-resolution mass spectrometer (Tokyo, Japan) in positive ion mode by using the matrix HEDS (bis-hydroxyethylsulfide) doped with sodium acetate. Acetonitrile was used after distillation over freshly ignited potassium carbonate.

\subsection{Synthesis and Characterization}

3.2.1. Synthesis of $2^{\prime}$-Deoxy-1'-(3-cyanomethyl-5-aryl)pyrazolyl-3', $5^{\prime}$-di-O-toluoyl- $\beta$-D-ribofuranose (3a-e) and 2'-deoxy-1'-(3-aryl-5-cyanomethyl)pyrazolyl-3', ,5'-di-O-toluoyl- $\beta$-D-ribofuranose (4a-e)

A solution of 3-cyanomethyl-5-arylpyrazole (1a-e, $15 \mathrm{mmol})$ in acetonitrile $(190 \mathrm{~mL})$ was added into a stirred mixture of sodium hydride $(22.5 \mathrm{mmol})$ in acetonitrile $(30 \mathrm{~mL})$ under a nitrogen atmosphere at $30-35{ }^{\circ} \mathrm{C}$, and continuously stirred for $30 \mathrm{~min}$. The reaction mixture was cooled to $0{ }^{\circ} \mathrm{C}$ and 1- $\alpha$-chloro-3,5-di-O-toluoyl-1,2-dideoxyribose $(2,15 \mathrm{mmol})$ was added and the contents stirred at $0{ }^{\circ} \mathrm{C}$ for 2.5 to $3 \mathrm{~h}$. The progress of the reaction was monitored by using silica gel TLC. On completion, the reaction mixture was poured over ice-cold water and extracted with ethyl acetate $(3 \times 50 \mathrm{~mL})$. The combined organic layer was washed with water $(2 \times 50 \mathrm{~mL})$, dried over anhydrous sodium sulfate, the solvent was removed under reduced pressure, and the residue thus obtained was column chromatographed over silica gel with ethyl acetate $(8-10 \%)$ in petroleum ether as the eluent to afford the pyrazolyl nucleosides $3 \mathbf{a}-\mathbf{e}$ and $\mathbf{4 a - e}$ in 31 to $35 \%$ and 24 to $29 \%$ yields, respectively.

2'-Deoxy-1'-(3-cyanomethyl-5-p-methylphenyl)pyrazolyl-3', 5'-di-O-toluoyl- $\beta$-D-ribofuranose (3a): Obtained as a white semisolid in a $34 \%$ yield. $[\alpha]_{\mathrm{D}}^{18}=-115.46^{\circ}\left(c 0.25, \mathrm{CHCl}_{3}\right) ; \mathrm{R}_{f}=0.53(20 \%$ ethyl acetate in petroleum ether); IR (Nujol): 2923, 2256 (CN), 1721 (COO), 1611, 1452, 1269, $1177 \mathrm{~cm}^{-1} ;{ }^{1} \mathrm{H} \mathrm{NMR}$ $\left(300 \mathrm{MHz} \mathrm{CDCl}_{3}\right): \delta 2.41\left(9 \mathrm{H}, \mathrm{s}, 3 \times \mathrm{Ar}_{-} \mathrm{CH}_{3}\right), 2.45-2.51\left(1 \mathrm{H}, \mathrm{m}, \mathrm{C}-2^{\prime} \mathrm{H}_{\alpha}\right), 3.45-3.52\left(1 \mathrm{H}, \mathrm{m}, \mathrm{C}-2^{\prime} \mathrm{H}_{\beta}\right)$, $3.65\left(2 \mathrm{H}, \mathrm{s}, \mathrm{CH}{ }_{2} \mathrm{CN}\right), 4.55-4.67\left(3 \mathrm{H}, \mathrm{m}, \mathrm{C}-4^{\prime} \mathrm{H}\right.$ and $\left.\mathrm{C}-5^{\prime} \mathrm{H}_{\alpha+\beta}\right), 5.86\left(1 \mathrm{H}, \mathrm{brs}, \mathrm{C}-3^{\prime} \mathrm{H}\right), 6.13(1 \mathrm{H}, \mathrm{t}$, $\left.J=6.1 \mathrm{~Hz}, \mathrm{C}-1^{\prime} \mathrm{H}\right), 6.33(1 \mathrm{H}, \mathrm{s}, \mathrm{C}-4 \mathrm{H}), 7.21-7.24(4 \mathrm{H}, \mathrm{brs}, 4 \times \mathrm{Ar}-\mathrm{H}), 7.28(2 \mathrm{H}, \mathrm{d}, J=7.8 \mathrm{~Hz}, \mathrm{Ar}-\mathrm{H}), 7.42$ $(2 \mathrm{H}, \mathrm{d}, J=7.8 \mathrm{~Hz}, \mathrm{Ar}-\mathrm{H}), 7.90(2 \mathrm{H}, \mathrm{d}, J=8.0 \mathrm{~Hz}, \mathrm{Ar}-\mathrm{H}), 8.00(2 \mathrm{H}, \mathrm{d}, J=8.0, \mathrm{Ar}-\mathrm{H}) ;{ }^{13} \mathrm{C} \mathrm{NMR}(75.5 \mathrm{MHz}$, $\left.\mathrm{CDCl}_{3}\right): \delta 15.6,19.3,19.7,19.7,34.6,62.4,73.9,80.6,84.5,103.8,115.2,124.4,124.7,125.2,127.0,127.1$, 127.1, 127.5, 127.7, 127.9, 137.2, 139.9, 142.1, 144.6, 141.7, 164.0, 164.3; HRMS (ESI): Calculated for $\mathrm{C}_{33} \mathrm{H}_{31} \mathrm{~N}_{3} \mathrm{O}_{5}[\mathrm{M}+\mathrm{Na}]^{+} 572.2156$, found $[\mathrm{M}+\mathrm{Na}]^{+} 572.2163$.

2'-Deoxy-1'-(3-cyanomethyl-5-p-methoxyphenyl)pyrazolyl-3',5'-di-O-toluoyl- $\beta$-D-ribofuranose (3b): Obtained as a semisolid in a $31 \%$ yield. $[\alpha]_{\mathrm{D}}^{18}=-119.46^{\circ}\left(c 0.25, \mathrm{CHCl}_{3}\right) ; \mathrm{R}_{f}=0.31(20 \%$ ethyl acetate in petroleum ether); IR (Nujol): 2926, 2256 (CN), 1724 (CO), 1610, 1505, 1254, $1178 \mathrm{~cm}^{-1} ;{ }^{1} \mathrm{H} \mathrm{NMR}\left(300 \mathrm{MHz}, \mathrm{CDCl}_{3}\right)$ : $\delta 2.40\left(6 \mathrm{H}, \mathrm{s}, 2 \times \mathrm{Ar}_{-} \mathrm{CH}_{3}\right), 2.46-2.50\left(1 \mathrm{H}, \mathrm{m}, \mathrm{C}-2^{\prime} \mathrm{H}_{\alpha}\right), 3.48-3.53\left(1 \mathrm{H}, \mathrm{m}, \mathrm{C}-2^{\prime} \mathrm{H}_{\beta}\right), 3.64\left(2 \mathrm{H}, \mathrm{s}, \mathrm{CH}{ }_{2} \mathrm{CN}\right)$, 
$3.84\left(3 \mathrm{H}, \mathrm{s}, \mathrm{OCH}_{3}\right), 4.54-4.62\left(3 \mathrm{H}, \mathrm{m}, \mathrm{C}-\mathrm{C}-4^{\prime} \mathrm{H} \& 5^{\prime} \mathrm{H}_{\alpha+\beta}\right), 5.87\left(1 \mathrm{H}, \mathrm{brs}, \mathrm{C}-3^{\prime} \mathrm{H}\right), 6.10\left(1 \mathrm{H}, \mathrm{brs}, \mathrm{C}-1^{\prime} \mathrm{H}\right)$, $6.30(1 \mathrm{H}, \mathrm{s}, \mathrm{C}-4 \mathrm{H}), 6.99(2 \mathrm{H}, \mathrm{d}, J=8.1, \mathrm{Ar}-\mathrm{H}), 7.21-7.23(4 \mathrm{H}, \mathrm{m}, \mathrm{Ar}-\mathrm{H}), 7.46(2 \mathrm{H}, \mathrm{d}, J=8.0 \mathrm{~Hz}, \mathrm{Ar}-\mathrm{H})$, $7.89(2 \mathrm{H}, \mathrm{d}, J=7.6 \mathrm{~Hz}, \mathrm{Ar}-\mathrm{H}), 7.99(2 \mathrm{H}, \mathrm{d}, J=7.6, \mathrm{Ar}-\mathrm{H}) ;{ }^{13} \mathrm{C}$ NMR $\left(75.5 \mathrm{MHz}, \mathrm{CDCl}_{3}\right): \delta 19.7,23.7$, 23.8, 38.7, 57.5, 66.5, 78.0, 84.7, 88.6, 107.8, 116.4, 119.3, 123.8, 128.9, 129.4, 131.1, 131.2, 131.8, 132.0, 132.6, 144.0, 145.8, 146.2, 148.5, 162.4, 168.1, 168.4; HRMS (ESI): Calculated for $\mathrm{C}_{33} \mathrm{H}_{31} \mathrm{~N}_{3} \mathrm{O}_{6}[\mathrm{M}+\mathrm{Na}]^{+}$ 588.2105 , found $[\mathrm{M}+\mathrm{Na}]^{+} 588.2127$.

2'-Deoxy-1'-(3-cyanomethyl-5-p-flurophenyl)pyrazolyl-3', $5^{\prime}$-di-O-toluoyl- $\beta$-D-ribofuranose (3c): Obtained as a white semisolid in a $35 \%$ yield. $[\alpha]_{\mathrm{D}}^{18}=-63.64^{\circ}\left(c 0.25, \mathrm{CHCl}_{3}\right) ; \mathrm{R}_{f}=0.54(20 \%$ ethyl acetate in petroleum ether); IR (Nujol): 2925, 2256 (CN), 1723 (COO), 1610, 1506, 1453, 1177, $1104 \mathrm{~cm}^{-1} ;{ }^{1} \mathrm{H}$ NMR $\left(300 \mathrm{MHz}, \mathrm{CDCl}_{3}\right): \delta 2.40\left(6 \mathrm{H}, \mathrm{s}, 2 \times \mathrm{Ar}^{-\mathrm{CH}_{3}}\right), 2.48-2.52\left(1 \mathrm{H}, \mathrm{m}, \mathrm{C}-2^{\prime} \mathrm{H}_{\alpha}\right), 3.50-3.54(1 \mathrm{H}, \mathrm{m}$, $\left.\mathrm{C}-2^{\prime} \mathrm{H}_{\beta}\right), 3.64\left(2 \mathrm{H}, \mathrm{s}, \mathrm{CH}_{2} \mathrm{CN}\right), 4.56-4.66\left(3 \mathrm{H}, \mathrm{m}, \mathrm{C}-4^{\prime} \mathrm{H} \& \mathrm{C}-5^{\prime} \mathrm{H}\right), 5.87\left(1 \mathrm{H}, \mathrm{brs}, \mathrm{C}-3^{\prime} \mathrm{H}\right), 6.05(1 \mathrm{H}, \mathrm{t}$, $\left.J=5.8 \mathrm{~Hz}, \mathrm{C}-1^{\prime} \mathrm{H}\right), 6.33(1 \mathrm{H}, \mathrm{s}, \mathrm{C}-4 \mathrm{H}), 7.13-7.24(6 \mathrm{H}, \mathrm{m}, \mathrm{Ar}-\mathrm{H}), 7.50-7.55(2 \mathrm{H}, \mathrm{m}, \mathrm{Ar}-\mathrm{H}), 7.90(2 \mathrm{H}, \mathrm{d}$, $J=7.9 \mathrm{~Hz}, \mathrm{Ar}-\mathrm{H}), 7.98(2 \mathrm{H}, \mathrm{d}, J=7.9 \mathrm{~Hz}, \mathrm{Ar}-\mathrm{H}) ;{ }^{13} \mathrm{C} \mathrm{NMR}\left(75.5 \mathrm{MHz}, \mathrm{CDCl}_{3}\right): \delta 17.5,21.5,21.6,36.4$, $64.2,75.7,82.6,86.3,106.1,115.9(\mathrm{~d}, J=22 \mathrm{~Hz}), 116.9,125.4,126.6,127.1,128.9,129.0,129.6,129.8,131.0$ (d, $J=8 \mathrm{~Hz}), 141.9,143.7,144.1,145.4,163.1(\mathrm{~d}, J=247 \mathrm{~Hz}), 165.9,166.1$; HRMS (ESI): Calculated for $\mathrm{C}_{32} \mathrm{H}_{28} \mathrm{~N}_{3} \mathrm{O}_{5} \mathrm{~F}[\mathrm{M}+\mathrm{Na}]^{+}$576.1905, found $[\mathrm{M}+\mathrm{Na}]^{+} 576.1916$.

2'-Deoxy-1'-(3-cyanomethyl-5-p-chlorophenyl)pyrazolyl-3',5'-di-O-toluoyl- $\beta$-D-ribofuranose (3d): Obtained as a yellow solid in a $32 \%$ yield. $[\alpha]_{\mathrm{D}}^{18}=-109.79^{\circ}\left(c 0.25, \mathrm{CHCl}_{3}\right) ; \mathrm{Mp}=114-115^{\circ} \mathrm{C} ; \mathrm{R}_{f}=0.52(20 \%$ ethyl acetate in petroleum ether); IR (Nujol): 2924, $2257(\mathrm{CN}), 1728$ (COO), 1610, 1494, 1177, $1108 \mathrm{~cm}^{-1}$; ${ }^{1} \mathrm{H}$ NMR $\left(300 \mathrm{MHz}, \mathrm{CDCl}_{3}\right): \delta 2.40\left(6 \mathrm{H}, \mathrm{s}, 2 \times \mathrm{Ar}^{-\mathrm{CH}_{3}}\right), 2.46-2.52\left(1 \mathrm{H}, \mathrm{m}, \mathrm{C}-2^{\prime} \mathrm{H}_{\alpha}\right), 3.50-3.57(1 \mathrm{H}, \mathrm{m}$, $\left.\mathrm{C}-2^{\prime} \mathrm{H}_{\beta}\right), 3.63\left(2 \mathrm{H}, \mathrm{s}, \mathrm{CH}_{2} \mathrm{CN}\right), 4.51-4.66\left(3 \mathrm{H}, \mathrm{m}, \mathrm{C}-4^{\prime} \mathrm{H} \& \mathrm{C}-5^{\prime} \mathrm{H}\right), 5.86-5.88\left(1 \mathrm{H}, \mathrm{m}, \mathrm{C}-3^{\prime} \mathrm{H}\right), 6.05(1 \mathrm{H}$, $\left.\mathrm{t}, J=6.1 \mathrm{~Hz}, \mathrm{C}-1^{\prime} \mathrm{H}\right), 6.35(1 \mathrm{H}, \mathrm{s}, \mathrm{C}-4 \mathrm{H}), 7.21-7.24(4 \mathrm{H}, \mathrm{m}, \mathrm{Ar}-\mathrm{H}), 7.43-7.50(4 \mathrm{H}, \mathrm{m}, \mathrm{Ar}-\mathrm{H}), 7.90(2 \mathrm{H}$, d, $J=7.9 \mathrm{~Hz}, \mathrm{Ar}-\mathrm{H}), 7.98(2 \mathrm{H}, \mathrm{d}, J=7.9 \mathrm{~Hz}, \mathrm{Ar}-\mathrm{H}) ;{ }^{13} \mathrm{C} \mathrm{NMR}\left(75.5 \mathrm{MHz}, \mathrm{CDCl}_{3}\right): \delta 17.4,21.5,21.6$, 36.4, 64.1, 75.6, 82.7, 86.4, 106.1, 116.9, 126.4, 126.6, 127.6, 128.9, 129.0, 129.1, 129.6, 129.7, 130.3, 135.3, 141.9, 143.7, 144.1, 145.2, 165.9, 166.1; HRMS (ESI): Calculated for $\mathrm{C}_{32} \mathrm{H}_{28} \mathrm{~N}_{3} \mathrm{O}_{5} \mathrm{Cl}[\mathrm{M}+\mathrm{Na}]^{+} 592.1610$, found $[\mathrm{M}+\mathrm{Na}]^{+} 592.1625$.

2'-Deoxy-1'-(3-cyanomethyl-5-p-bromophenyl)pyrazolyl-3', 5'-di-O-toluoyl- $\beta$-D-ribofuranose (3e): Obtained as a white solid in a $35 \%$ yield. $[\alpha]_{\mathrm{D}}^{18}=-96.35^{\circ}\left(c 0.25, \mathrm{CHCl}_{3}\right) ; \mathrm{Mp}=118-110{ }^{\circ} \mathrm{C} ; \mathrm{R}_{f}=0.47(20 \%$ ethyl acetate in petroleum ether); IR (Nujol): 2921, 2255 (CN), 1714 (COO), 1610, 1463, 1267, 1176, $1101 \mathrm{~cm}^{-1}$; ${ }^{1} \mathrm{H}$ NMR $\left(300 \mathrm{MHz}, \mathrm{CDCl}_{3}\right): \delta 2.41\left(6 \mathrm{H}, \mathrm{s}, 2 \times \mathrm{Ar}^{-\mathrm{CH}_{3}}\right), 2.46-2.54\left(1 \mathrm{H}, \mathrm{m}, \mathrm{C}-2^{\prime} \mathrm{H}_{\alpha}\right), 3.48-3.57(1 \mathrm{H}, \mathrm{m}$, $\left.\mathrm{C}-2^{\prime} \mathrm{H}_{\beta}\right), 3.64\left(2 \mathrm{H}, \mathrm{s}, \mathrm{CH}_{2} \mathrm{CN}\right), 4.51-4.66\left(3 \mathrm{H}, \mathrm{m}, \mathrm{C}-4^{\prime} \mathrm{H} \& \mathrm{C}-5^{\prime} \mathrm{H}\right), 5.85-5.87\left(1 \mathrm{H}, \mathrm{m}, \mathrm{C}-3^{\prime} \mathrm{H}\right), 6.05(1 \mathrm{H}, \mathrm{t}$, $\left.J=6.1 \mathrm{~Hz}, \mathrm{C}-1^{\prime} \mathrm{H}\right), 6.35(1 \mathrm{H}, \mathrm{s}, \mathrm{C}-4 \mathrm{H}), 7.22(4 \mathrm{H}, \mathrm{d}, J=7.9 \mathrm{~Hz}, \mathrm{Ar}-\mathrm{H}), 7.42(2 \mathrm{H}, \mathrm{d}, J=8.3 \mathrm{~Hz}, \mathrm{Ar}-\mathrm{H})$, $7.60(2 \mathrm{H}, \mathrm{d}, J=8.3 \mathrm{~Hz}, \mathrm{Ar}-\mathrm{H}), 7.90(2 \mathrm{H}, \mathrm{d}, J=8.0 \mathrm{~Hz}, \mathrm{Ar}-\mathrm{H}), 7.98(2 \mathrm{H}, \mathrm{d}, J=8.0 \mathrm{~Hz}, \mathrm{Ar}-\mathrm{H}) ;{ }^{13} \mathrm{C}$ NMR $\left(75.5 \mathrm{MHz}, \mathrm{CDCl}_{3}\right): \delta 17.8,21.9,21.9,36.8,64.5,76.0,83.1,86.8,106.5,117.3,123.9,127.0,127.4,128.5$, 129.3, 129.4, 130.0, 130.1, 130.9, 132.4, 142.3, 144.0, 144.4, 145.6, 166.2, 166.5; HRMS (ESI): Calculated for $\mathrm{C}_{32} \mathrm{H}_{28} \mathrm{~N}_{3} \mathrm{O}_{5} \mathrm{Br}[\mathrm{M}+\mathrm{Na}]^{+}$636.1105, found $[\mathrm{M}+\mathrm{Na}]^{+}$636.1113.

2'-Deoxy-1'-(3-p-methylphenyl-5-cyanomethyl)pyrazolyl-3',5'-di-O-toluoyl- $\beta$-D-ribofuranose (4a): Obtained as a white semisolid in a $26 \%$ yield. $[\alpha]_{\mathrm{D}}^{18}=+25.10^{\circ}\left(c 0.25, \mathrm{CHCl}_{3}\right) ; \mathrm{R}_{f}=0.36(20 \%$ ethyl acetate in petroleum ether); IR (Nujol): 2923, $2256(\mathrm{CN}), 1721$ (COO), 1611, 1452, 1269, $1177 \mathrm{~cm}^{-1},{ }^{1} \mathrm{H}$ NMR $\left(300 \mathrm{MHz}, \mathrm{CDCl}_{3}\right): \delta 2.31,2.38$ and $2.42\left(9 \mathrm{H}, 3 \mathrm{~s}, 3 \mathrm{H}\right.$ each, $\left.3 \times \mathrm{Ar}^{-\mathrm{CH}_{3}}\right), 2.65-2.70\left(1 \mathrm{H}, \mathrm{m}, \mathrm{C}-2^{\prime} \mathrm{H}_{\alpha}\right)$, 3.74-3.78 $\left(1 \mathrm{H}, \mathrm{m}, \mathrm{C}-2^{\prime} \mathrm{H}_{\beta}\right), 3.91\left(2 \mathrm{H}, \mathrm{s}, \mathrm{CH}_{2} \mathrm{CN}\right), 4.36\left(1 \mathrm{H}, \mathrm{q}, J=6.3 \mathrm{~Hz}, \mathrm{C}-4^{\prime} \mathrm{H}\right), 4.54-4.60\left(2 \mathrm{H}, \mathrm{m}, \mathrm{C}-5^{\prime} \mathrm{H}\right)$, 5.85-5.87 (1H, m, C-3'H), $6.17\left(1 \mathrm{H}, \mathrm{t}, J=5.7 \mathrm{~Hz}, \mathrm{C}-1^{\prime} \mathrm{H}\right), 6.59(1 \mathrm{H}, \mathrm{s}, \mathrm{C}-4 \mathrm{H}), 7.03(2 \mathrm{H}, \mathrm{d}, J=8.0 \mathrm{~Hz}$, Ar-H), $7.18(2 \mathrm{H}, \mathrm{d}, J=7.9 \mathrm{~Hz}, \mathrm{Ar}-\mathrm{H}), 7.26(2 \mathrm{H}, \mathrm{d}, J=8.1 \mathrm{~Hz}, \mathrm{Ar}-\mathrm{H}), 7.63(2 \mathrm{H}, \mathrm{d}, J=8.0 \mathrm{~Hz}, \mathrm{Ar}-\mathrm{H}), 7.83$ $(2 \mathrm{H}, \mathrm{d}, J=8.1 \mathrm{~Hz}, \mathrm{Ar}-\mathrm{H}), 7.95(2 \mathrm{H}, \mathrm{d}, J=8.0 \mathrm{~Hz}, \mathrm{Ar}-\mathrm{H}) ;{ }^{13} \mathrm{C} \mathrm{NMR}\left(75.5 \mathrm{MHz}, \mathrm{CDCl}_{3}\right): \delta 15.28,21.2$, 21.5, 21.6, 36.0, 63.6, 75.0, 83.2, 87.0, 105.3, 115.3, 125.6, 126.6, 126.7, 128.9, 129.0, 129.1, 129.2, 129.6, 129.7, 133.0, 137.9, 143.5, 144.2, 151.0, 165.9, 166.0; HRMS (ESI): Calculated for $\mathrm{C}_{33} \mathrm{H}_{31} \mathrm{~N}_{3} \mathrm{O}_{5}[\mathrm{M}+\mathrm{Na}]^{+}$ 572.2156, found $[\mathrm{M}+\mathrm{Na}]^{+} 572.2155$. 
2'-Deoxy-1'-(3-p-methoxyphenyl-5-cyanomethyl)pyrazolyl-3', $5^{\prime}$-di-O-toluoyl- $\beta$-D-ribofuranose (4b): Obtained as a white solid in a $24 \%$ yield. $[\alpha]_{\mathrm{D}}^{18}=+42.05^{\circ}\left(c 0.25, \mathrm{CHCl}_{3}\right) ; \mathrm{Mp}=88-90{ }^{\circ} \mathrm{C} ; \mathrm{R}_{f}=0.24(20 \%$ ethyl acetate in petroleum ether); IR (Nujol): 2924, 2256 (CN), 1724 (COO), 1610, 1456, 1270, 1178 $\mathrm{cm}^{-1} ;{ }^{1} \mathrm{H}$ NMR $\left(300 \mathrm{MHz}, \mathrm{CDCl}_{3}\right): \delta 2.32$ and $2.42\left(6 \mathrm{H}, 2 \mathrm{~s}, 3 \mathrm{H}\right.$ each, $\left.2 \times \mathrm{Ar}_{-} \mathrm{CH}_{3}\right), 2.65-2.69\left(1 \mathrm{H}, \mathrm{m}, \mathrm{C}-2^{\prime} \mathrm{H}_{\alpha}\right)$, 3.73-3.77 (1H, m, C-2' $\left.\mathrm{H}_{\beta}\right), 3.83\left(3 \mathrm{H}, \mathrm{s}, \mathrm{Ar}-\mathrm{OCH}_{3}\right), 3.90\left(2 \mathrm{H}, \mathrm{s}, \mathrm{CH}_{2} \mathrm{CN}\right), 4.34-4.37\left(1 \mathrm{H}, \mathrm{m}, \mathrm{C}-4^{\prime} \mathrm{H}\right)$, 4.56-4.59 (2H, m, C-5'H), 5.87 (1H, brs, C-3'H), 6.16 (1H, brs, C-1'H), $6.55(1 \mathrm{H}, \mathrm{s}, \mathrm{C}-4 \mathrm{H}), 6.91(2 \mathrm{H}, \mathrm{d}$, $J=7.6 \mathrm{~Hz}, \mathrm{Ar}-\mathrm{H}), 7.05(2 \mathrm{H}, \mathrm{d}, J=7.3 \mathrm{~Hz}, \mathrm{Ar}-\mathrm{H}), 7.26(2 \mathrm{H}, \mathrm{d}, J=6.9 \mathrm{~Hz}, \mathrm{Ar}-\mathrm{H}), 7.67(2 \mathrm{H}, \mathrm{d}, J=7.7 \mathrm{~Hz}$, Ar-H), $7.84(2 \mathrm{H}, \mathrm{d}, J=7.3 \mathrm{~Hz}, \mathrm{Ar}-\mathrm{H}), 7.96(2 \mathrm{H}, \mathrm{d}, J=7.4 \mathrm{~Hz}, \mathrm{Ar}-\mathrm{H}) ;{ }^{13} \mathrm{C}\left(75.5 \mathrm{MHz}, \mathrm{CDCl}_{3}\right): \delta 15.2$, 21.5, 21.6, 36.0, 55.2, 63.6, 75.0, 83.1, 86.9, 105.0, 113.9, 115.4, 125.2, 126.6, 126.9, 128.9, 129.1, 129.5, 129.6, $129.7,133.0,143.5,144.1,150.8,159.6,165.8,165.9$; HRMS (ESI): Calculated for $\mathrm{C}_{33} \mathrm{H}_{31} \mathrm{~N}_{3} \mathrm{O}_{6}[\mathrm{M}+\mathrm{Na}]^{+}$ 588.2105 , found $[\mathrm{M}+\mathrm{Na}]^{+} 588.2134$.

2'-Deoxy-1'-(3-p-flurophenyl-5-cyanomethyl)pyrazolyl-3', $5^{\prime}$-di-O-toluoyl- $\beta$-D-ribofuranose (4c): Obtained as a white semisolid in a $25 \%$ yield. $[\alpha]_{\mathrm{D}}^{18}=+14.07^{\circ}\left(c 0.25, \mathrm{CHCl}_{3}\right) ; \mathrm{R}_{f}=0.47(20 \%$ ethyl acetate in petroleum ether); IR (Nujol): 2925, 2257 (CN), 1725 (COO), 1610,1522,1443,1272, 1178, $1102 \mathrm{~cm}^{-1} ;{ }^{1} \mathrm{H}$ $\mathrm{NMR}\left(300 \mathrm{MHz}, \mathrm{CDCl}_{3}\right): \delta 2.31$, and $2.42\left(6 \mathrm{H}, 2 \mathrm{~s}, 3 \mathrm{H}\right.$ each, $\left.2 \times \mathrm{Ar}_{-} \mathrm{CH}_{3}\right), 2.66-2.70\left(1 \mathrm{H}, \mathrm{m}, \mathrm{C}-2^{\prime} \mathrm{H}_{\alpha}\right)$, 3.73-3.77 (1H, m, C-2' $\left.\mathrm{H}_{\beta}\right), 3.92\left(2 \mathrm{H}, \mathrm{s}, \mathrm{CH}_{2} \mathrm{CN}\right), 4.33-4.35\left(1 \mathrm{H}, \mathrm{m}, \mathrm{C}-4^{\prime} \mathrm{H}\right), 4.58-4.63\left(2 \mathrm{H}, \mathrm{m}, \mathrm{C}-5^{\prime} \mathrm{H}\right)$, $5.87\left(1 \mathrm{H}\right.$, brs, $\left.\mathrm{C}-3^{\prime} \mathrm{H}\right), 6.17\left(1 \mathrm{H}, \mathrm{t}, J=5.1 \mathrm{~Hz}, \mathrm{C}-1^{\prime} \mathrm{H}\right), 6.56(1 \mathrm{H}, \mathrm{s}, \mathrm{C}-4 \mathrm{H}), 7.02-7.08(4 \mathrm{H}, \mathrm{m}, \mathrm{Ar}-\mathrm{H}), 7.26$ $(2 \mathrm{H}, \mathrm{d}, J=7.9 \mathrm{~Hz}, \mathrm{Ar}), 7.67-7.71(2 \mathrm{H}, \mathrm{m}, \mathrm{Ar}-\mathrm{H}), 7.83(2 \mathrm{H}, \mathrm{d}, J=8.0 \mathrm{~Hz}, \mathrm{Ar}-\mathrm{H}), 7.96(2 \mathrm{H}, \mathrm{d}, J=8.0$ $\mathrm{Hz}, \mathrm{Ar}-\mathrm{H}) ;{ }^{13} \mathrm{C}$ NMR $\left(75.5 \mathrm{MHz}, \mathrm{CDCl}_{3}\right): \delta 17.7,23.9,24.0,38.5,66.0,77.3,85.7,89.5,107.7,117.8$ $(\mathrm{d}, J=22 \mathrm{~Hz}), 128.0,129.0(\mathrm{~d}, J=7.5 \mathrm{~Hz}), 129.7,129.8,131.0,131.4,131.6,132.0,132.1,135.8,146.0,146.7$, $152.5,165.4(\mathrm{~d}, \mathrm{~J}=250 \mathrm{~Hz}), 168.2,168.4$; HRMS (ESI): Calculated for $\mathrm{C}_{32} \mathrm{H}_{28} \mathrm{~N}_{3} \mathrm{O}_{5} \mathrm{~F}[\mathrm{M}+\mathrm{Na}]^{+}$576.1905, found $[\mathrm{M}+\mathrm{Na}]^{+} 576.1915$.

2'-Deoxy-1'-(3-p-chlorophenyl-5-cyanomethyl)pyrazolyl-3',5'-di-O-toluoyl- $\beta$-D-ribofuranose (4d): Obtained as a yellow solid in a $24 \%$ yield. $[\alpha]_{\mathrm{D}}^{18}=+34.06^{\circ}\left(c 0.25, \mathrm{CHCl}_{3}\right) ; \mathrm{Mp}=109-110{ }^{\circ} \mathrm{C} ; \mathrm{R}_{f}=0.40(20 \%$ ethyl acetate in petroleum ether); IR (nujol): 2924, 2257 (CN), 1716 (COO), 1459, 1377, 1176, $\mathrm{cm}^{-1}$; ${ }^{1} \mathrm{H} \mathrm{NMR}$ $\left(300 \mathrm{MHz}_{\mathrm{CDCl}}\right): \delta 2.33$ and $2.45\left(6 \mathrm{H}, 2 \mathrm{~s}, 3 \mathrm{H}\right.$ each, $2 \times \mathrm{Ar}^{\left.-\mathrm{CH}_{3}\right)}, 2.65-2.74\left(1 \mathrm{H}, \mathrm{m}, \mathrm{C}-2^{\prime} \mathrm{H}_{\alpha}\right), 3.74-3.85$ $\left(1 \mathrm{H}, \mathrm{m}, \mathrm{C}-2^{\prime} \mathrm{H}_{\beta}\right), 3.94\left(2 \mathrm{H}, \mathrm{s}, \mathrm{CH}_{2} \mathrm{CN}\right), 4.33-4.38\left(1 \mathrm{H}, \mathrm{m}, \mathrm{C}-4^{\prime} \mathrm{H}\right), 4.57-4.65\left(2 \mathrm{H}, \mathrm{m}, \mathrm{C}-5^{\prime} \mathrm{H}\right), 5.88-5.90$ $\left(1 \mathrm{H}, \mathrm{m}, \mathrm{C}-3^{\prime} \mathrm{H}\right), 6.19\left(1 \mathrm{H}, \mathrm{t}, J=5.8 \mathrm{~Hz}, \mathrm{C}-1^{\prime} \mathrm{H}\right), 6.59(1 \mathrm{H}, \mathrm{s}, \mathrm{C}-4 \mathrm{H}), 7.04(2 \mathrm{H}, \mathrm{d}, J=7.9 \mathrm{~Hz}, \mathrm{Ar}-\mathrm{H}), 7.29$ $(2 \mathrm{H}, \mathrm{d}, J=8.0 \mathrm{~Hz}, \mathrm{Ar}-\mathrm{H}), 7.35(2 \mathrm{H}, \mathrm{d}, J=8.4 \mathrm{~Hz}, \mathrm{Ar}-\mathrm{H}), 7.66(2 \mathrm{H}, \mathrm{d}, J=8.4 \mathrm{~Hz}, \mathrm{Ar}-\mathrm{H}), 7.82(2 \mathrm{H}, \mathrm{d}$, $J=8.1 \mathrm{~Hz}, \mathrm{Ar}-\mathrm{H}), 7.98(2 \mathrm{H}, \mathrm{d}, J=8.0 \mathrm{~Hz}, \mathrm{Ar}-\mathrm{H}) ;{ }^{13} \mathrm{C} \mathrm{NMR}\left(75.5 \mathrm{MHz}, \mathrm{CDCl}_{3}\right): \delta 15.7,21.9,22.0,36.5$, 63.9, 75.3, 83.8, 87.6, 105.8, 116.5, 127.0, 127.3, 129.1, 129.4, 129.6, 130.0, 130.1, 130.2, 131.3, 133.9, 134.3, 144.1, 144.7, 150.3, 166.2, 166.4; HRMS (ESI): Calculated for $\mathrm{C}_{32} \mathrm{H}_{28} \mathrm{~N}_{3} \mathrm{O}_{5} \mathrm{Cl}[\mathrm{M}+\mathrm{Na}]^{+} 592.1610$, found $[\mathrm{M}+\mathrm{Na}]^{+} 592.1621$.

2'-Deoxy-1'-(3-p-bromophenyl-5-cyanomethyl-)pyrazolyl-3',5'-di-O-toluoyl- $\beta$-D-ribofuranose (4e): Obtained as a white solid in a $29 \%$ yield. $[\alpha]_{\mathrm{D}}^{18}=+39.82^{\circ}\left(c 0.25, \mathrm{CHCl}_{3}\right) ; \mathrm{Mp}=118-120{ }^{\circ} \mathrm{C} ; \mathrm{R}_{f}=0.25(20 \%$ ethyl acetate in petroleum ether); IR (Nujol): 2923, 2255 (CN), 1716 (COO), 1611, 1464, 1270, 1177 cm ${ }^{-1} ;{ }^{1} \mathrm{H}$

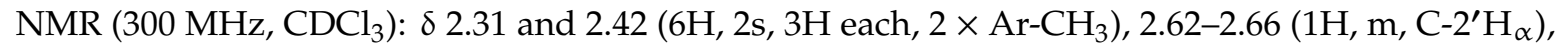
3.79-3.82 (1H, m, C-2' $\left.\mathrm{H}_{\beta}\right), 3.92\left(2 \mathrm{H}, \mathrm{s}, \mathrm{CH}_{2} \mathrm{CN}\right), 4.32-4.36\left(1 \mathrm{H}, \mathrm{m}, \mathrm{C}-4^{\prime} \mathrm{H}\right), 4.56-4.62\left(2 \mathrm{H}, \mathrm{m}, \mathrm{C}-5^{\prime} \mathrm{H}\right)$, 5.86-5.88 (1H, m, C-3' $\mathrm{H}), 6.17\left(1 \mathrm{H}, \mathrm{t}, J=5.7 \mathrm{~Hz}, \mathrm{C}-1^{\prime} \mathrm{H}\right), 6.57(1 \mathrm{H}, \mathrm{s}, \mathrm{C}-4 \mathrm{H}), 7.03(2 \mathrm{H}, \mathrm{d}, J=7.9 \mathrm{~Hz}$, Ar-H), $7.26(2 \mathrm{H}, \mathrm{d}, J=7.9 \mathrm{~Hz}, \mathrm{Ar}-\mathrm{H}), 7.48(2 \mathrm{H}, \mathrm{d}, J=8.3 \mathrm{~Hz}, \mathrm{Ar}-\mathrm{H}), 7.57(2 \mathrm{H}, \mathrm{d}, J=8.4 \mathrm{~Hz}, \mathrm{Ar}-\mathrm{H}), 7.80$ $(2 \mathrm{H}, \mathrm{d}, J=8.0 \mathrm{~Hz}, \mathrm{Ar}-\mathrm{H}), 7.96(2 \mathrm{H}, \mathrm{d}, J=8.0 \mathrm{~Hz}, \mathrm{Ar}-\mathrm{H}) ;{ }^{13} \mathrm{C} \mathrm{NMR}\left(75.5 \mathrm{MHz}, \mathrm{CDCl}_{3}\right): \delta 15.2,21.5,21.6$, 36.0, 63.4, 74.8, 83.3, 87.1, 105.3, 115.3, 122.0, 126.5, 126.6, 127.2, 128.9, 129.1, 129.5, 129.7, 131.3, 131.6, 133.5, 143.6, 144.2, 149.8, 166.0, 166.1; HRMS (ESI): Calculated for $\mathrm{C}_{32} \mathrm{H}_{28} \mathrm{~N}_{3} \mathrm{O}_{5} \mathrm{Br}[\mathrm{M}+\mathrm{Na}]^{+} 636.1105$, found $[\mathrm{M}+\mathrm{Na}]^{+} 636.1116$.

3.2.2. Synthesis of $2^{\prime}$-Deoxy-1'-(3-cyanomethyl-5-aryl)pyrazolyl- $\beta$-D-ribofuranose (5a-e) and 2'-deoxy-1'-(3-aryl-5-cyanomethyl)pyrazolyl- $\beta$-D-ribofuranose (6a-e)

$2^{\prime}$-Deoxy-1' -(3-cyanomethyl-5-aryl)pyrazolyl-3',5'-di-O-toluoyl- $\beta$-D-ribofuranose (3a-e) or $2^{\prime}$-deoxy-1'-(3-aryl-5-cyanomethyl)pyrazolyl-3', ,5'-di-O-toluoyl- $\beta$-D-ribofuranose (4a-e, $\left.1.3 \mathrm{mmol}\right)$ 
was suspended in dry methanol $(18 \mathrm{~mL})$, then a mixture of $\mathrm{NaOMe}$ and $\mathrm{MeOH}(10 \mathrm{~mL}, 1: 3, \mathrm{v} / \mathrm{v}) \mathrm{was}$ added to the resulting solution, and the contents stirred at room temperature for 3-4 h. The progress of the reaction was monitored by using silica gel TLC. On completion, $\mathrm{NH}_{4} \mathrm{Cl}$ was added to the reaction mixture to adjust the $\mathrm{pH}$ to 8 . One-third of the solvent was removed under reduced pressure and the reaction mixture was poured in water and extracted with ethyl acetate $(3 \times 30 \mathrm{~mL})$, the organic layer was dried over anhydrous sodium sulfate, the solvent was removed under reduced pressure, and the residue thus obtained was subjected to column chromatography over silica gel with methanol (2-2.5\%) in chloroform as the eluent to afford $2^{\prime}$-deoxy-1'-(3-cyanomethyl-5-aryl)pyrazolyl- $\beta$-D-ribofuranose (5a-e) and $2^{\prime}$-deoxy-1'-(3-aryl-5-cyanomethyl)pyrazolyl- $\beta$-D-ribofuranose (6a-e).

2'-Deoxy-1'-(3-cyanomethyl-5-p-methylphenyl)pyrazolyl- $\beta$-D-ribofuranose (5a): Obtained as a white semisolid in a $63 \%$ yield. $[\alpha]_{\mathrm{D}}^{30}=-87.56^{\circ}($ c $0.25, \mathrm{MeOH}) ; \mathrm{R}_{f}=0.3(7 \%$ methanol in chloroform); IR (Nujol): 3391 (OH), 2936, 2258 (CN), 1613, 1506, 1456, 1253, 1180, $1055 \mathrm{~cm}^{-1}$; ${ }^{1} \mathrm{H}$ NMR (300 MHz, $\left.\mathrm{CDCl}_{3}\right): \delta 2.10-2.18\left(1 \mathrm{H}, \mathrm{m}, \mathrm{C}-2^{\prime} \mathrm{H}_{\alpha}\right), 2.39\left(3 \mathrm{H}, \mathrm{s}, \mathrm{Ar}-\mathrm{CH}_{3}\right), 2.87-2.91\left(1 \mathrm{H}, \mathrm{m}, \mathrm{C}-2^{\prime} \mathrm{H}_{\beta}\right), 3.53$ (1H, brs, C-5' $\left.\mathrm{H}_{\alpha}\right), 3.61\left(1 \mathrm{H}\right.$, brs, C-5' $\left.\mathrm{H}_{\beta}\right), 3.84(1 \mathrm{H}$, brs, C-4' $\mathrm{H}), 3.90\left(2 \mathrm{H}, \mathrm{s}, \mathrm{CH} \mathrm{C}_{2} \mathrm{CN}\right), 4.44\left(1 \mathrm{H}, \mathrm{brs}, \mathrm{C}-3^{\prime} \mathrm{H}\right)$, $4.49(1 \mathrm{H}$, brs, C-3'OH), $5.13(1 \mathrm{H}$, brs, C-5'OH), $5.99(1 \mathrm{H}$, brs, C-1'H), $6.30(1 \mathrm{H}, \mathrm{s}, \mathrm{C}-4 \mathrm{H}), 7.29(2 \mathrm{H}, \mathrm{d}$, $J=7.3, \mathrm{Ar}-\mathrm{H}), 7.40(2 \mathrm{H}, \mathrm{d}, J=7.7 \mathrm{~Hz}, \mathrm{Ar}-\mathrm{H}) ;{ }^{13} \mathrm{C} \mathrm{NMR}\left(75.5 \mathrm{MHz}, \mathrm{CDCl}_{3}\right): \delta 17.3,21.1,39.9,63.0,71.6$, 86.0, 88.3, 105.3, 117.7, 126.5, 128.9, 129.5, 138.8, 142.0, 145.9; HRMS (ESI): Calculated for $\mathrm{C}_{17} \mathrm{H}_{19} \mathrm{~N}_{3} \mathrm{O}_{3}$ $[\mathrm{M}+\mathrm{Na}]^{+} 336.1319$, found $[\mathrm{M}+\mathrm{Na}]^{+} 336.1288$.

2'-Deoxy-1'-(3-cyanomethyl-5-p-methoxyphenyl)pyrazolyl- $\beta$-D-ribofuranose (5b): Obtained as a white semisolid in a $66 \%$ yield. $[\alpha]_{\mathrm{D}}^{30}=-94.35^{\circ}(c 0.25, \mathrm{MeOH}) ; \mathrm{R}_{f}=0.48$ (7\% methanol in chloroform); IR (Nujol): 3391 (OH), 2932, 2254 (CN), 1612, 1510, 1457, 1253, 1183, $1056 \mathrm{~cm}^{-1}$; ${ }^{1} \mathrm{H}$ NMR $(300 \mathrm{MHz}$, DMSO-d $\left.d_{6}\right): \delta 2.17-2.21\left(1 \mathrm{H}, \mathrm{m}, \mathrm{C}-2^{\prime} \mathrm{H}_{\alpha}\right), 2.89-2.93\left(1 \mathrm{H}, \mathrm{m}, \mathrm{C}-2^{\prime} \mathrm{H}_{\beta}\right), 3.57-3.61\left(1 \mathrm{H}, \mathrm{m}, \mathrm{C}-5^{\prime} \mathrm{H}_{\alpha}\right), 3.65-3.70$

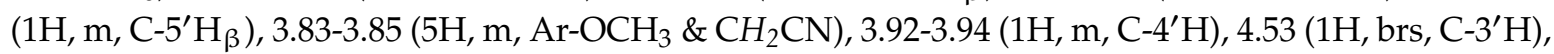
$4.64\left(1 \mathrm{H}, \mathrm{t}, J=6.6, \mathrm{C}-5^{\prime} \mathrm{OH}\right), 5.09\left(1 \mathrm{H}, \mathrm{d}, J=4.1 \mathrm{~Hz}, \mathrm{C}-3^{\prime} \mathrm{OH}\right), 6.02\left(1 \mathrm{H}, \mathrm{t}, J=6.3 \mathrm{~Hz}, \mathrm{C}-1^{\prime} \mathrm{H}\right), 6.26(1 \mathrm{H}, \mathrm{s}$, $\mathrm{C}-4 \mathrm{H}), 7.00(2 \mathrm{H}, \mathrm{d}, J=8.6 \mathrm{~Hz}, \mathrm{Ar}-\mathrm{H}), 7.42(2 \mathrm{H}, \mathrm{d}, J=8.6 \mathrm{~Hz}, \mathrm{Ar}-\mathrm{H}) ;{ }^{13} \mathrm{C}$ NMR $\left(75.5 \mathrm{MHz}, \mathrm{DMSO}-d_{6}\right): \delta$ 17.3, 39.9, 55.3, 63.1, 71.8, 86.0, 88.4, 105.1, 114.3, 117.4, 121.5, 130.3, 141.8, 145.8, 160.1; HRMS (ESI): Calculated $\mathrm{C}_{17} \mathrm{H}_{19} \mathrm{~N}_{3} \mathrm{O}_{4}[\mathrm{M}+\mathrm{Na}]^{+} 352.1268$, found $[\mathrm{M}+\mathrm{Na}]^{+} 352.1248$.

2'-Deoxy-1'-(3-cyanomethyl-5-p-fluorophenyl)pyrazolyl- $\beta$-D-ribofuranose (5c): Obtained as a semisolid in a $63 \%$ yield. $[\alpha]_{\mathrm{D}}^{30}=-153.52^{\circ}($ c $0.25, \mathrm{MeOH}) ; \mathrm{R}_{f}=0.41$ (7\% methanol in chloroform); IR (Nujol): 3393 (OH), 2932, 2256 (CN), 1611, 1508, 1451, 1255, 1182, $1051 \mathrm{~cm}^{-1} ;{ }^{1} \mathrm{H}$ NMR (300 MHz, DMSO-d 6 ): $\delta$ 2.18-2.22 (1H, m, C-2' $\left.\mathrm{H}_{\alpha}\right), 2.91-2.95\left(1 \mathrm{H}, \mathrm{m}, \mathrm{C}-2^{\prime} \mathrm{H}_{\beta}\right), 3.61\left(1 \mathrm{H}, \mathrm{brs}, \mathrm{C}-5^{\prime} \mathrm{H}_{\alpha}\right), 3.65\left(1 \mathrm{H}, \mathrm{brs}, \mathrm{C}-5^{\prime} \mathrm{H}_{\beta}\right)$, $3.87(2 \mathrm{H}, \mathrm{s}, \mathrm{CH} \mathrm{CN} \mathrm{CN}), 3.93\left(1 \mathrm{H}, \mathrm{brs}, \mathrm{C}-4^{\prime} \mathrm{H}\right), 4.53\left(2 \mathrm{H}, \mathrm{brs}, \mathrm{C}-3^{\prime} \mathrm{H}\right.$ and $\left.\mathrm{OH}\right), 5.08\left(1 \mathrm{H}, \mathrm{brs}, \mathrm{C}-5^{\prime} \mathrm{OH}\right), 5.97$ $\left(1 \mathrm{H}\right.$, brs, $\left.\mathrm{C}-1^{\prime} \mathrm{H}\right), 6.32(1 \mathrm{H}, \mathrm{s}, \mathrm{C}-4 \mathrm{H}), 7.22$ (2H, brs, Ar-H), 7.53 (2H, brs, Ar-H); ${ }^{13} \mathrm{C}$ NMR $(75.5 \mathrm{MHz}$, DMSO- $\left.d_{6}\right): \delta 17.3,39.9,63.0,71.7,86.1,88.4,105.7,115.9(\mathrm{~d}, J=22 \mathrm{~Hz}), 117.3,125.6,131.1(\mathrm{~d}, J=8 \mathrm{~Hz})$, $141.9,144.9,162.8\left(\mathrm{~d}, \mathrm{~J}=246 \mathrm{~Hz}\right.$ ); HRMS (ESI): Calculated for $\mathrm{C}_{16} \mathrm{H}_{16} \mathrm{~N}_{3} \mathrm{O}_{3} \mathrm{~F}[\mathrm{M}+\mathrm{Na}]^{+} 340.1068$, found $[\mathrm{M}+\mathrm{Na}]^{+} 340.1058$.

2'-Deoxy-1'-(3-cyanomethyl-5-p-chlorophenyl)pyrazolyl- $\beta$-D-ribofuranose (5d): Obtained as a semisolid in a $63 \%$ yield. $[\alpha]_{\mathrm{D}}^{30}=-124.74^{\circ}(c 0.25, \mathrm{MeOH}) ; \mathrm{R}_{f}=0.46$ (7\% methanol in chloroform); IR (Nujol): 3393 $(\mathrm{OH}), 2932,2256(\mathrm{CN}), 1611,1498,1451,1254,1181,1048 \mathrm{~cm}^{-1} ;{ }^{1} \mathrm{H}$ NMR (300 MHz, DMSO- $\left.d_{6}\right): \delta$ 2.22-2.24 (1H, m, C-2' $\left.\mathrm{H}_{\alpha}\right), 2.92-2.96\left(1 \mathrm{H}, \mathrm{m}, \mathrm{C}-2^{\prime} \mathrm{H}_{\beta}\right), 3.59-3.61\left(1 \mathrm{H}, \mathrm{m}, \mathrm{C}-5^{\prime} \mathrm{H}_{\alpha}\right), 3.68-3.72(1 \mathrm{H}, \mathrm{m}$, C-5' $\left.\mathrm{H}_{\beta}\right), 3.85\left(2 \mathrm{H}\right.$, brs, $\left.\mathrm{CH}_{2} \mathrm{CN}\right), 3.97(1 \mathrm{H}$, brs, C-4' $\mathrm{H}), 4.57$ (2H, brs, C-3' $\mathrm{H}$ and $\left.\mathrm{OH}\right), 5.09$ (1H, brs, C-5'OH), $6.00\left(1 \mathrm{H}, \mathrm{brs}, \mathrm{C}-1^{\prime} \mathrm{H}\right), 6.34(1 \mathrm{H}, \mathrm{s}, \mathrm{C}-4 \mathrm{H}), 7.24(2 \mathrm{H}, \mathrm{d}, J=7.5 \mathrm{~Hz}, \mathrm{Ar}-\mathrm{H}), 7.87(2 \mathrm{H}, \mathrm{d}, J=8.5 \mathrm{~Hz}$, Ar-H); ${ }^{13} \mathrm{C}\left(75.5 \mathrm{MHz}\right.$, DMSO- $\left.d_{6}\right): \delta 17.3,39.9,63.1,71.8,86.2,88.5,105.7,117.8,127.8,129.3,130.4$, 134.7, 141.9, 144.7; HRMS (ESI): Calculated for $\mathrm{C}_{16} \mathrm{H}_{16} \mathrm{~N}_{3} \mathrm{O}_{3} \mathrm{Cl}[\mathrm{M}+\mathrm{Na}]^{+} 356.0772$, found [M + Na] ${ }^{+}$ 356.0770 .

2'-Deoxy-1'-(3-cyanomethyl-5-p-bromophenyl)pyrazolyl- $\beta$-D-ribofuranose (5e): Obtained as a semisolid in a $64 \%$ yield. $[\alpha]_{\mathrm{D}}^{30}=-105.15^{\circ}(c 0.25, \mathrm{MeOH}) ; \mathrm{R}_{f}=0.53$ (7\% methanol in chloroform); IR (Nujol): 3387 (OH), 2935, 2249 (CN), 1612, 1504, 1454, 1248, 1179, $1048 \mathrm{~cm}^{-1} ;{ }^{1} \mathrm{H}$ NMR (300 MHz, DMSO- $\left.d_{6}\right): \delta$ 
2.20-2.24 (1H, m, C-2' $\left.\mathrm{H}_{\alpha}\right), 2.90-2.96\left(1 \mathrm{H}, \mathrm{m}, \mathrm{C}-2^{\prime} \mathrm{H}_{\beta}\right), 3.59-3.61\left(1 \mathrm{H}, \mathrm{m}, \mathrm{C}-5^{\prime} \mathrm{H}_{\alpha}\right), 3.68-3.72(1 \mathrm{H}, \mathrm{m}$, $\left.\mathrm{C}-5^{\prime} \mathrm{H}_{\beta}\right), 3.85\left(2 \mathrm{H}\right.$, brs, $\left.\mathrm{CH}_{2} \mathrm{CN}\right), 3.97\left(1 \mathrm{H}, \mathrm{brs}, \mathrm{C}-4^{\prime} \mathrm{H}\right), 4.56\left(2 \mathrm{H}, \mathrm{brs}, \mathrm{C}-3^{\prime} \mathrm{H}\right.$ \& OH $), 5.07(1 \mathrm{H}$, brs, $\left.\mathrm{C}-5^{\prime} \mathrm{OH}\right), 6.00\left(1 \mathrm{H}, \mathrm{t}, J=6.06 \mathrm{~Hz}, \mathrm{C}-1^{\prime} \mathrm{H}\right), 6.34(1 \mathrm{H}, \mathrm{s}, \mathrm{C}-4 \mathrm{H}), 7.42(2 \mathrm{H}, \mathrm{d}, J=8.1 \mathrm{~Hz}, \mathrm{Ar}-\mathrm{H}), 7.61(2 \mathrm{H}$, $\mathrm{d}, J=8.0 \mathrm{~Hz}, \mathrm{Ar}-\mathrm{H}) ;{ }^{13} \mathrm{C}\left(75.5 \mathrm{MHz}\right.$, DMSO- $\left.d_{6}\right): \delta 16.5,39.0,62.2,71.0,85.3,87.6,104.8,116.3,122.2$, 128.2, 129.8, 131.0, 141.1, 143.9; HRMS (ESI): Calculated for $\mathrm{C}_{16} \mathrm{H}_{16} \mathrm{~N}_{3} \mathrm{O}_{3} \mathrm{Br}[\mathrm{M}+\mathrm{Na}]^{+} 400.0267$, found $[\mathrm{M}+\mathrm{Na}]^{+} 400.0232$.

2'-Deoxy-1'-(5-cyanomethyl-3-p-methylphenyl)pyrazolyl- $\beta$-D-ribofuranose (6a): Obtained as a white semisolid in a $61 \%$ yield. $[\alpha]_{\mathrm{D}}^{30}=+34.78^{\circ}($ c $0.25, \mathrm{MeOH}) ; \mathrm{R}_{f}=0.3$ (7\% methanol in chloroform); IR (Nujol): 3392 (OH), 2934, 2258 (CN), 1611, 1504, 1452, 1254, 1179, $1051 \mathrm{~cm}^{-1}$; ${ }^{1} \mathrm{H}$ NMR (300 MHz, $\left.\mathrm{CDCl}_{3}\right): \delta 2.23-2.27\left(1 \mathrm{H}, \mathrm{m}, \mathrm{C}-2^{\prime} \mathrm{H}_{\alpha}\right), 2.31\left(3 \mathrm{H}, \mathrm{s}, \mathrm{Ph}-\mathrm{CH}_{3}\right), 2.77-2.83\left(1 \mathrm{H}, \mathrm{m}, \mathrm{C}-2^{\prime} \mathrm{H}_{\beta}\right), 3.36-3.40(1 \mathrm{H}$, $\left.\mathrm{m}, \mathrm{C}-5^{\prime} \mathrm{H}_{\alpha}\right), 3.49-3.51\left(1 \mathrm{H}, \mathrm{m}, \mathrm{C}^{\prime} \mathrm{H}_{\beta}\right), 3.82-3.86\left(1 \mathrm{H}, \mathrm{m}, \mathrm{C}-4^{\prime} \mathrm{H}\right), 4.32\left(2 \mathrm{H}, \mathrm{s}, \mathrm{CH}_{2} \mathrm{CN}\right), 4.46(1 \mathrm{H}, \mathrm{brs}$, $\left.\mathrm{C}-3^{\prime} \mathrm{H}\right), 4.86\left(1 \mathrm{H}, \mathrm{t}, J=5.4 \mathrm{~Hz}, \mathrm{C}-5^{\prime} \mathrm{OH}\right), 5.29\left(1 \mathrm{H}, \mathrm{d}, J=4.0 \mathrm{~Hz}, \mathrm{C}-3^{\prime} \mathrm{OH}\right), 6.13\left(1 \mathrm{H}, \mathrm{t}, J=5.9 \mathrm{~Hz}, \mathrm{C}-1^{\prime} \mathrm{H}\right)$, $6.72(1 \mathrm{H}, \mathrm{s}, \mathrm{C}-4 \mathrm{H}), 7.22$ and $7.69(4 \mathrm{H}, 2 \mathrm{~d}, 2 \mathrm{H}$ each, $J=7.8 \mathrm{~Hz}, \& 7.9 \mathrm{~Hz}, \mathrm{ArH}) ;{ }^{13} \mathrm{C}$ NMR $(75.5 \mathrm{MHz}$, DMSO- $\left.d_{6}\right): \delta 14.6,21.1,39.8,62.6,71.4,86.5,88.4,104.0,117.5,125.5,129.6,130.1,135.0,137.7,150.2$; HRMS (ESI): Calculated for $\mathrm{C}_{17} \mathrm{H}_{19} \mathrm{~N}_{3} \mathrm{O}_{3}[\mathrm{M}+\mathrm{Na}]^{+} 336.1319$, found $[\mathrm{M}+\mathrm{Na}]^{+} 336.1290$.

2'-Deoxy-1'-(5-cyanomethyl-3-p-methoxyphenyl)pyrazolyl- $\beta$-D-ribofuranose (6b): Obtained as a semisolid in a $62 \%$ yield. $[\alpha]_{\mathrm{D}}^{30}=+56.37^{\circ}(c 0.25, \mathrm{MeOH}) ; \mathrm{R}_{f}=0.39$ (7\% methanol in chloroform); IR (Nujol): $3385(\mathrm{OH}), 2937,2258(\mathrm{CN}), 1610,1501,1448,1251,1183,1053 \mathrm{~cm}^{-1} ;{ }^{1} \mathrm{H}$ NMR (300 MHz, DMSO- $d_{6}$ ): 反 2.40-2.42 $\left(1 \mathrm{H}, \mathrm{m}, \mathrm{C}-2^{\prime} \mathrm{H}_{\alpha}\right), 2.94-2.98\left(1 \mathrm{H}, \mathrm{m}, \mathrm{C}-2^{\prime} \mathrm{H}_{\beta}\right), 3.60-3.64\left(1 \mathrm{H}, \mathrm{m}, \mathrm{C}-5^{\prime} \mathrm{H}_{\alpha}\right), 3.72-3.76(1 \mathrm{H}$, $\left.\mathrm{m}, \mathrm{C}-5^{\prime} \mathrm{H}_{\beta}\right), 3.81\left(3 \mathrm{H}, \mathrm{s}, \mathrm{Ph}-\mathrm{OCH}_{3}\right), 3.87\left(1 \mathrm{H}, \mathrm{brs}, \mathrm{C}-4^{\prime} \mathrm{H}\right), 4.08\left(2 \mathrm{H}, \mathrm{s}, \mathrm{CH}_{2} \mathrm{CN}\right), 4.61\left(1 \mathrm{H}\right.$, brs, $\left.\mathrm{C}-3^{\prime} \mathrm{H}\right)$, 5.01-5.05 (2H, m, C-5' OH \& C-3'OH), $6.19\left(1 \mathrm{H}, \mathrm{t}, J=6.0 \mathrm{~Hz}, \mathrm{C}-1^{\prime} \mathrm{H}\right), 6.56(1 \mathrm{H}, \mathrm{s}, \mathrm{C}-4 \mathrm{H}), 6.91$ and 7.66 $(4 \mathrm{H}, 2 \mathrm{~d}, 2 \mathrm{H}$ each, $J=8.4 \mathrm{~Hz}$ and $8.4 \mathrm{~Hz}, \mathrm{Ar}-\mathrm{H}) ;{ }^{13} \mathrm{C}$ NMR $\left(75.5 \mathrm{MHz}\right.$, DMSO- $\left.d_{6}\right): \delta 14.9,39.9,55.1$, 63.2, 71.9, 86.9, 88.9, 103.6, 114.0, 116.1, 126.7, 129.3, 133.7, 150.7, 159.6; HRMS (ESI): Calculated for $\mathrm{C}_{17} \mathrm{H}_{19} \mathrm{~N}_{3} \mathrm{O}_{4}[\mathrm{M}+\mathrm{Na}]^{+} 352.1268$, found $[\mathrm{M}+\mathrm{Na}]^{+} 352.1237$.

2'-Deoxy-1'-(5-cyanomethyl-3-p-fluorophenyl)pyrazolyl- $\beta$-D-ribofuranose (6c): Obtained as a semisolid in a $59 \%$ yield. $[\alpha]_{\mathrm{D}}^{30}=+47.97^{\circ}(c 0.25, \mathrm{MeOH}) ; \mathrm{R}_{f}=0.36$ (7\% methanol in chloroform); IR (Nujol): 3391 (OH), 2928, 2249 (CN), 1610, 1503, 1448, 1252, 1179, $1055 \mathrm{~cm}^{-1} ;{ }^{1} \mathrm{H} \mathrm{NMR}\left(300 \mathrm{MHz}, \mathrm{CDCl}_{3}\right): \delta 2.24-2.28$ $\left(1 \mathrm{H}, \mathrm{m}, \mathrm{C}-2^{\prime} \mathrm{H}_{\alpha}\right), 2.85-2.89\left(1 \mathrm{H}, \mathrm{m}, \mathrm{C}-2^{\prime} \mathrm{H}_{\beta}\right), 3.39-3.41\left(1 \mathrm{H}, \mathrm{m}, \mathrm{C}-5^{\prime} \mathrm{H}_{\alpha}\right), 3.48-3.54\left(1 \mathrm{H}, \mathrm{m}, \mathrm{C}-5^{\prime} \mathrm{H}_{\beta}\right)$, 3.82-3.87 (1H, m, C- $\left.4^{\prime} \mathrm{H}\right), 4.33\left(2 \mathrm{H}, \mathrm{s}, \mathrm{CH}_{2} \mathrm{CN}\right), 4.46\left(1 \mathrm{H}, \mathrm{brs}, \mathrm{C}-3^{\prime} \mathrm{H}\right), 4.81\left(1 \mathrm{H}, \mathrm{t}, J=5.4 \mathrm{~Hz}, \mathrm{C}-5^{\prime} \mathrm{OH}\right)$, $5.27\left(1 \mathrm{H}, \mathrm{d}, J=4.0 \mathrm{~Hz}, \mathrm{C}-3^{\prime} \mathrm{OH}\right), 6.16\left(1 \mathrm{H}, \mathrm{t}, J=6.0 \mathrm{~Hz}, \mathrm{C}-1^{\prime} \mathrm{H}\right), 6.76(1 \mathrm{H}, \mathrm{s}, \mathrm{C}-4 \mathrm{H}), 7.24(2 \mathrm{H}, \mathrm{t}, J=7.6 \mathrm{~Hz})$, $7.83(2 \mathrm{H}, \mathrm{q}, J=5.3 \mathrm{~Hz}, \mathrm{Ar}-\mathrm{H}) ;{ }^{13} \mathrm{C}$ NMR $\left(75.5 \mathrm{MHz}, \mathrm{DMSO}-d_{6}\right): \delta 15.2,40.3,63.1,71.9,87.0,89.0,104.7$, $116.5(\mathrm{~d}, J=22 \mathrm{~Hz}), 117.9,128.1(\mathrm{~d}, J=8 \mathrm{~Hz}), 130.0,135.8,149.8,162.9$ (d, $J=243 \mathrm{~Hz}) ;$ HRMS (ESI): Calculated for $\mathrm{C}_{16} \mathrm{H}_{16} \mathrm{~N}_{3} \mathrm{O}_{3} \mathrm{~F}[\mathrm{M}+\mathrm{Na}]^{+} 340.1068$, found $[\mathrm{M}+\mathrm{Na}]^{+} 340.1038$.

2'-Deoxy-1'-(3-p-chlorophenyl-5-cyanomethyl-)pyrazolyl- $\beta$-D-ribofuranose (6d): Obtained as a semisolid in a $61 \%$ yield. $[\alpha]_{\mathrm{D}}^{30}=+37.58^{\circ}(c 0.25, \mathrm{MeOH}) ; \mathrm{R}_{f}=0.36$ (7\% methanol in chloroform); IR (Nujol): $3387(\mathrm{OH}), 2935,2249$ (CN), 1612, 1504, 1454, 1248, 1179, $1048 \mathrm{~cm}^{-1} ;{ }^{1} \mathrm{H}$ NMR $\left(300 \mathrm{MHz}, \mathrm{CD}_{3} \mathrm{CN}\right): \delta$ 2.36-2.43 $\left(1 \mathrm{H}, \mathrm{m}, \mathrm{C}-2^{\prime} \mathrm{H}_{\alpha}\right), 2.88-2.96\left(1 \mathrm{H}, \mathrm{m}, \mathrm{C}-2^{\prime} \mathrm{H}_{\beta}\right), 3.43\left(1 \mathrm{H}, \mathrm{brs}, \mathrm{C}-4^{\prime} \mathrm{H}\right), 3.56-3.63\left(1 \mathrm{H}, \mathrm{m}, \mathrm{C}-5^{\prime} \mathrm{H}_{\alpha}\right)$, 3.69-3.73 (1H, m, C-5' $\left.\mathrm{H}_{\beta}\right), 4.05-4.11\left(4 \mathrm{H}, \mathrm{m}, \mathrm{CH}_{2} \mathrm{CN}, \mathrm{C}-3^{\prime} \mathrm{H} \& \mathrm{C}-5^{\prime} \mathrm{OH}\right), 4.62\left(1 \mathrm{H}, \mathrm{brs}, \mathrm{C}-3^{\prime} \mathrm{OH}\right), 6.17$ $\left(1 \mathrm{H}, \mathrm{t}, J=6.1 \mathrm{~Hz}, \mathrm{C}-1^{\prime} \mathrm{H}\right), 6.72(1 \mathrm{H}, \mathrm{s}, \mathrm{C}-4 \mathrm{H}), 7.44(2 \mathrm{H}, \mathrm{d}, J=8.5 \mathrm{~Hz}, \mathrm{Ar}-\mathrm{H}), 7.77(2 \mathrm{H}, \mathrm{d}, J=8.5 \mathrm{~Hz}$, Ar-H); ${ }^{13} \mathrm{C}$ NMR $\left(75.5 \mathrm{MHz}, \mathrm{CD}_{3} \mathrm{CN}\right): \delta 14.4,39.9,62.6,71.7,86.6,88.6,103.7,116.9,126.6,128.5,130.9$, 133.1, 134.6, 149.6; HRMS (ESI): Calculated for $\mathrm{C}_{16} \mathrm{H}_{16} \mathrm{~N}_{3} \mathrm{O}_{3} \mathrm{Cl}[\mathrm{M}+\mathrm{Na}]^{+} 356.0772$, found [M + Na] ${ }^{+}$ 356.0757.

$2^{\prime}$-Deoxy-1'-(3-p-bromophenyl-5-cyanomethyl)pyrazolyl- $\beta$-D-ribofuranose (6e): Obtained as a white solid in a $63 \%$ yield. $[\alpha]_{\mathrm{D}}^{30}=+50.37^{\circ}(c 0.25, \mathrm{MeOH}) ; \mathrm{Mp}=74-75^{\circ} \mathrm{C} ; \mathrm{R}_{f}=0.39$ (7\% methanol in chloroform); IR (Nujol): 3385 (OH), 2937, 2258 (CN), 1610, 1501, 1448, 1251, 1183, $1053 \mathrm{~cm}^{-1}$; ${ }^{1} \mathrm{H}$ NMR (300 MHz, DMSO- $\left.d_{6}\right): \delta 2.34-2.39\left(1 \mathrm{H}, \mathrm{m}, \mathrm{C}-2^{\prime} \mathrm{H}_{\alpha}\right), 2.92-2.98\left(1 \mathrm{H}, \mathrm{m}, \mathrm{C}-2^{\prime} \mathrm{H}_{\beta}\right), 3.47\left(1 \mathrm{H}, \mathrm{brs}, \mathrm{C}-4^{\prime} \mathrm{H}\right), 3.58(1 \mathrm{H}, \mathrm{brs}$, C-5 $\left.{ }^{\prime} \mathrm{H}_{\alpha}\right), 3.95\left(1 \mathrm{H}\right.$, brs, $\left.\mathrm{C}-5^{\prime} \mathrm{H}_{\beta}\right), 4.24\left(2 \mathrm{H}, \mathrm{s}, \mathrm{CH}_{2} \mathrm{CN}\right), 4.51\left(1 \mathrm{H}, \mathrm{brs}, 3^{\prime} \mathrm{H}\right), 4.77\left(1 \mathrm{H}\right.$, brs, $\left.\mathrm{C}-3^{\prime} \mathrm{OH}\right), 5.20$ $\left(1 \mathrm{H}, \mathrm{brs}, \mathrm{C}-5^{\prime} \mathrm{OH}\right), 6.18\left(1 \mathrm{H}, \mathrm{s}, \mathrm{C}-1^{\prime} \mathrm{H}\right), 6.71(1 \mathrm{H}, \mathrm{s}, \mathrm{C}-4 \mathrm{H}), 7.55(2 \mathrm{H}, \mathrm{brs}, \mathrm{Ar}-\mathrm{H}), 7.69(2 \mathrm{H}, \mathrm{brs}, \mathrm{Ar}-\mathrm{H})$; 
${ }^{13} \mathrm{C}$ NMR $\left(75.5 \mathrm{MHz}\right.$, DMSO-d $\left.d_{6}\right): \delta 14.9,39.7,62.7,71.4,86.9,88.6,104.3,116.8,121.5,127.3,129.2$, 131.7, 134.9, 149.2; HRMS (ESI): Calculated for $\mathrm{C}_{16} \mathrm{H}_{16} \mathrm{~N}_{3} \mathrm{O}_{3} \mathrm{Br}[\mathrm{M}+\mathrm{Na}]^{+} 400.0267$, found [M + Na $]^{+}$ 400.0234 .

\subsection{NCI-60 Human Tumor Cell Line Screen}

Details of the methodology are described at http://dtp.nci.nih.gov/branches/btb/ivclsp.html. Briefly, the panel was organized into nine subpanels representing a diverse histology: leukemia, melanoma, and cancers of the lung, colon, kidney, ovary, breast, prostate, and central nervous system. The cells were grown in supplemented RPM1 1640 medium for $24 \mathrm{~h}$. For the five dose study, the test compounds were dissolved in DMSO and incubated with cells at five concentrations with 10-fold dilutions, the highest being $10^{-4} \mathrm{M}$ and the others being $10^{-5}, 10^{-6}, 10^{-7}$, and $10^{-8} \mathrm{M}$. The assay was terminated by the addition of cold trichloroacetic acid, and the cells were fixed and stained with sulforhodamine B. The bound stain was solubilized, and the absorbance was read on an automated plate reader. The cytostatic parameter, which determines the $50 \%$ growth inhibition $\left(\mathrm{GI}_{50}\right)$ of the tumor cells, was calculated from time zero, control growth, and the absorbance at the five concentration levels. The cytotoxic parameter, lethal concentration $\left(\mathrm{LC}_{50}\right.$ is the concentration of a drug resulting in a $50 \%$ reduction in the measured protein at the end of the drug treatment when compared to that at the beginning), indicating a net loss of cells following the treatment, was calculated as the average of two independent experiments.

\section{Conclusions}

We successfully achieved the synthesis of twenty novel pyrazolyl nucleosides $\mathbf{3 a}-\mathbf{e}, \mathbf{4} \mathbf{a}-\mathbf{e}, \mathbf{5 a}-\mathbf{e}$, and $\mathbf{6 a - e}$, which have been characterized in detail by using various NMR spectroscopic techniques such as ${ }^{1} \mathrm{H}$ NMR, ${ }^{13} \mathrm{C}$ NMR, NOESY, HMBC, etc. The pyrazolyl nucleosides 5a-e and $\mathbf{6 a}-\mathbf{e}$ were screened for anticancer activities on 60 human tumor cell lines, and we identified one compound $6 \mathbf{e}$, which showed good activity against 39 cancer cell lines and showed significant inhibition against lung cancer cell line Hop-92 (GI $9.3 \mu \mathrm{M})$ and breast cancer cell line HS 578T ( $\left.\mathrm{GI}_{50} 3.0 \mu \mathrm{M}\right)$. Our studies have demonstrated the potential of newly synthesized pyrazolyl-nucleosides that will be pursued further.

Supplementary Materials: The following are available online at http://www.mdpi.com/1420-3049/24/21/3922/s1, Scanned copies of the NMR and IR spectra of selected compounds, as appropriate are given in the "Supplementary Information".

Author Contributions: Y.Y., K.K. and D.S. synthesized the compounds; V.K. and S.V.M. compiled the NCI-60 activity; A.J. interpreted the NMR data and drafted the manuscript; J.W., C.L., A.K.P. and V.S.P. planned and designed the whole study and finalized the manuscript.

Funding: Please add: This research received no external funding; See the "Acknowledgements Section" for details.

Acknowledgments: We would like to thank the Department of Biotechnology (DBT, Govt. of India, New Delhi), the University of Delhi (DU, India), and the Nucleic Acid Center (NAC, University of Southern Denmark, Odense, Denmark) for their financial assistance. The authors are grateful to Carl-Erik Olsen, University of Copenhagen, Denmark for carrying out extensive NMR spectral studies and to Eric De Clercq, Rega Institute for Medical Research, K.U. Leuven, Belgium for carrying out the antiviral evaluation studies.

Conflicts of Interest: The authors declare no conflict of interest.

\section{References}

1. Yates, M.K.; Seley-Radtke, K.L. The evolution of antiviral nucleoside analogues: A review for chemists and non-chemists. Part II: Complex modifications to the nucleoside scaffold. Antivir. Res. 2019, 162, 5-21. [CrossRef] [PubMed]

2. Singh, S.; Bhattarai, D.; Veeraswamy, G.; Choi, Y.; Lee, K. Nucleosides with modified sugar ring: Synthesis and biological activities. Curr. Org. Chem. 2016, 20, 856-897. [CrossRef] 
3. Zhou, L.; Zhang, H.; Tao, S.; Ehteshami, M.; Cho, J.H.; McBrayer, T.R.; Tharnish, P.; Whitaker, T.; Amblard, F.; Coats, S.J.; et al. Synthesis and evaluation of 2,6-modified purine 2'-C-methyl ribonucleosides as inhibitors of HCV replication. ACS Med. Chem. Lett. 2016, 7, 17-22. [CrossRef] [PubMed]

4. De Clercq, E. Milestones in the discovery of antiviral agents: Nucleosides and nucleotides. Acta Pharm. Sin. B. 2012, 2, 535-548. [CrossRef]

5. Zhou, C.; Chattopadhyaya, J. The synthesis of therapeutic locked nucleos(t)ides. Curr. Opin. Drug. Discov. Devel. 2009, 12, 876-898. [CrossRef]

6. Wan, J.; Xia, Y.; Liu, Y.; Wang, M.; Rocchi, P.; Yao, J.; Qu, F.; Neyts, J.; Iovanna, J.L.; Peng, L. Discovery of novel arylethynyltriazole ribonucleosides with selective and effective antiviral and antiproloferative activity. J. Med. Chem. 2009, 52, 1144-1155. [CrossRef]

7. Chu, C.K.; Baker, D.C. (Eds.) Nucleosides and Nucleotides as Antitumor and Antiviral Agents; Plenum Press: New York, NY, USA, 1993; pp. 245-264.

8. Prakash, T.P. An overview of sugar-modified oligonucleosides for antisense therapeutics. Chem. Biodivers. 2011, 8, 1616-1641. [CrossRef]

9. Yu, R.Z.; Grundy, J.S.; Geary, R.S. Clinical pharmacokinetics of second generation antisense oligonucleotides. Expert Opin. Drug Metab. Toxicol. 2013, 9, 169-182. [CrossRef]

10. Watts, J.K. Locked nucleic acid: Tighter is different. Chem. Commun. 2013, 49, 5618-5620. [CrossRef]

11. Mitsuya, H.; Broder, S. Inhibition of the in vitro infectivity and cytopathic effect of human T-lymphotrophic virus type III/lymphadenopathy-associated virus (HTLV-III/LAV) by 2',3'-dideoxynucleosides. Proc. Natl. Acad. Sci. USA 1986, 83, 1911-1915. [CrossRef]

12. Len, C.; Postel, D. Synthesis of 2', $3^{\prime}$-didehydro-2', $3^{\prime}$-dideoxynucleosides via nucleoside route. Curr. Org. Synth. 2006, 3, 261-281. [CrossRef]

13. Len, C.; Mackenzie, G. Synthesis of 2',3'-didehydro-2', $3^{\prime}$-dideoxynucleosides having variations at either or both of the 2'- and 3'-positions. Tetrahedron 2006, 62, 9085-9107. [CrossRef]

14. Len, C.; Mondon, M.; Lebreton, J. Synthesis of cyclonucleosides having a C-C bridge. Tetrahedron 2008, 64, 7453-7475. [CrossRef]

15. Wengel, J. Synthesis of 3'-C-and 4'-C-branched oligodeoxynucleotides and the development of locked nucleic acid (LNA). Acc. Chem. Res. 1999, 32, 301-310. [CrossRef]

16. Petersen, M.; Wengel, J. LNA: A versatile tool for therapeutics and genomics. Trends Biotechnol. 2003, 21, 74-81. [CrossRef]

17. Kalra, N.; Babu, B.R.; Parmar, V.S.; Wengel, J. Conformationally controlled high-affinity targeting of RNA or DNA by novel 2'-amino-DNA/LNA mixmers and pyrenyl-functionalized 2'-amino-DNA. Org. Biomol. Chem. 2004, 2, 2885-2887. [CrossRef]

18. Babu, B.R.; Hrdlicka, P.J.; McKenzie, C.J.; Wengel, J. Optimizing DNA targeting using N,N-bis(2-pyridylmethyl)-|?-alanyl 2'-amino-LNA. Chem. Commun. 2005, 1705-1707. [CrossRef]

19. Hrdlicka, P.J.; Jepsen, J.S.; Nielsen, C.; Wengel, J. Synthesis and biological evaluation of nucleobase-modified analogs of the anticancer compounds 3'-C-ethynyluridine (EUrd) and 3'-C-ethynylcytidine (ECyd). Bioorg. Med. Chem. 2005, 13, 1249-1260. [CrossRef]

20. Prakash, T.P.; Prhavc, M.; Eldrup, A.B.; Cook, P.D.; Carroll, S.S.; Olsen, D.B.; Stahlhut, M.W.; Tomassini, J.E.; MacCoss, M.; Galloway, S.M.; et al. Synthesis and evaluation of S-acyl-2-thioethyl esters of modified nucleoside 5'-monophosphates as inhibitors of hepatitis C virus RNA replication. J. Med. Chem. 2005, 48, 1199-1210. [CrossRef]

21. Sharma, V.K.; Watts, J.K. Oligonucleotide therapeutics: Chemistry, delivery and clinical progress. Future Med. Chem. 2015, 7, 2221-2242. [CrossRef]

22. Reid, G.; Wielinga, P.; Zelcer, N.; de Haas, M.; van Deemter, L.; Wijnholds, J.; Balzarini, J.; Borst, P. Characterization of the transport of nucleoside analog drugs by the human multidrug resistance proteins MRP4 and MRP5. Mol. Pharmacol. 2003, 63, 1094-1103. [CrossRef] [PubMed]

23. Parmar, V.S.; Jain, S.C.; Jha, A.; Kumar, N.; Kumar, A.; Vats, A.; Jha, H.N.; Mukherjee, S.; Singh, S.K.; Jennings, K.R.; et al. Synthetic and mass spectral fragmentation studies on trisubstituted 2H-pyran-2-ones and comparative EIMS behavior of biologically active 3,5-disubstituted pyrazoles and isoxazoles. Indian J. Chem. 1997, 36B, 872-879. [CrossRef]

24. Sharma, V.K.; Sharma, R.K.; Singh, P.K.; Singh, S.K. An engrossing history of azidothymidine. Immun. Endoc. Metab. Agents Med. Chem. 2015, 15, 168-175. [CrossRef] 
25. Sidwell, R.W.; Huffman, J.H.; Khare, G.P.; Allen, L.B.; Witkowski, J.T.; Robins, R.K. Broad-spectrum antiviral activity of virazole: 1- $\beta$-D-ribofuranosyl-1,2,4-triazole-3-carboxamide. Science 1972, 177, 705-706. [CrossRef] [PubMed]

26. Storer, R.; Ashton, C.J.; Baxter, A.D.; Hann, M.M.; Marr, C.L.P.; Mason, A.M.; Mo, C.L.; Myers, P.L.; Noble, S.A.; Penn, C.R.; et al. The synthesis and antiviral activity of 4-fluoro-1- $\beta$-D-ribofuranosyl-1H-pyrazole-3-carboxamide. Nucleosides Nucleotides 1999, 18, 203-216. [CrossRef] [PubMed]

27. Balzarini, J.; Kang, G.J.; Dalal, M.; Herdewijn, P.; De Clercq, E.; Broder, S.; Johns, D.G. The anti-HTLV-III (anti-HIV) and cytotoxic activity of $2^{\prime}, 3^{\prime}$-didehydro-2', $3^{\prime}$-dideoxynucleosides: A comparison with their parental 2',3'-dideoxyribonucleosides. Mol. Pharmacol. 1987, 32, 162-167.

28. Hamamoto, Y.; Nakashima, H.; Matsui, T.; Mateuda, A.; Ueda, T.; Yamamoto, N. Inhibitory effect of 2',3'-didehydro-2',3'-dideoxynucleosides on infectivity, cytopathic effects, and replication of human immunodeficiency vírus. Antimicrob. Agents Chemother. 1987, 31, 907-910. [CrossRef]

29. Lin, T.S.; Guo, J.Y.; Schinazi, R.F.; Chu, C.K.; Xiang, J.N.; Prusoff, W.H. Synthesis and antiviral activity of various 3'azido analogues of pyrimidine deoxyribonucleosides against Human Immunodeficiency Virus (HIV-1, HTLV-III/LAV). J. Med. Chem. 1988, 31, 336-340. [CrossRef]

30. Haneishi, T.; Okazaki, T.; Hata, T.; Tamura, C.; Nomura, M.; Naito, A.; Seki, I.; Arai, M. Oxazinomycin, a new carbon-linked nucleoside antibiotic. J. Antibiot. 1971, 24, 797-799. [CrossRef]

31. Sweeney, M.J.; Davis, F.A.; Gutowski, G.E.; Hamill, R.L.; Hoffman, D.H.; Poore, G.A. Experimental antitumor activity of pyrazomycin. Cancer Res. 1973, 33, 2619-2623.

32. Harusawa, S.; Matsuda, C.; Araki, L.; Kurihara, T. Efficient and $\beta$-steroselective synthesis of pyrazole C-nucleosides. Synthesis 2006, 793-798. [CrossRef]

33. Nishimura, H.; Mayama, M.; Komatsu, Y.; Kato, H.; Shimaoka, N.; Tanaka, Y. Showdomycin, a new antibiotic from a Streptomyces Sp. J. Antibiot. Ser. A. 1964, 17, 148-155.

34. Michalik, D.; Peseke, K. Syntheses of pyrazole iso-C-nucleosides. J. Carbohydr. Chem. 2000, 19, $1049-1057$. [CrossRef]

35. Hori, M.; Ito, E.; Takita, T.; Koyama, G.; Takeuchi, T.; Umezawa, H. A new antibiotics, Formycin. J. Antibiot. Ser. A. 1964, 17, 96-99.

36. Zhou, J.; Yang, M.; Akdag, A.; Wang, H.; Schneller, S.W. Carbocyclic 4'-epi-formycin. Tetrahedron 2008, 64, 433-438. [CrossRef]

37. Moriyama, K.; Suzuki, T.; Negishi, K.; Graci, J.D.; Thompson, C.N.; Cameron, C.E.; Watanabe, M. Effects of introduction of hydrophobic group on ribavirin base on mutation induction and anti-RNA viral activity. J. Med. Chem. 2008, 51, 159-166. [CrossRef]

38. Manfredini, S.; Baraldi, P.G.; Bazzanini, R.; Durini, E.; Vertuani, S.; Pani, A.; Marceddu, T.; Demontis, F.; Vargiu, L.; La Calla, P. Pyrazole related nucleosides 5. Synthesis and biological activity of 2'-deoxy-2',3'-dideoxy- and acyclo-analogues of 4-iodo-1- $\beta$-D-ribofuranosyl-3-carboxymethyl pyrazole (IPCAR). Nucleosides Nucleotides Nucleic Acids 2000, 19, 705-722. [CrossRef]

39. Rauter, A.P.; Figueiredo, J.A.; Ismael, M.I.; Justino, J. Synthesis of new pseudo-C-nucleosides containing pyrazole rings in their structure. J. Carbohydr. Chem. 2004, 23, 513-528. [CrossRef]

40. Zhang, J.; Visser, F.; King, K.M.; Baldwin, S.A.; Young, J.D.; Cass, C.E. The role of nucleoside transporters in cancer chemotherapy with nucleoside drugs. Cancer Metastasis Rev. 2007, 26, 85-110. [CrossRef]

41. Podgorska, M.; Kocbuch, K.; Pawelczyk, T. Recent advances in studies on biochemical and structural properties of equilibrative and concentrative nucleoside transporter. Acta Biochim. Pol. 2005, 52, 749-758.

42. Uhlmann, E.; Peyman, A. Antisense oligonucleotides: A new therapeutic principle. Chem. Rev. 1990, 90, 544-584. [CrossRef]

43. Dhimitruka, I.; Santa Lucia, J., Jr. Efficient preparation of 2-deoxy-3,5-di-O-p-toluoyl- $\alpha$-D-ribofuranosyl chloride. Synlett 2004, 335-337. [CrossRef]

44. Chenon, M.T.; Coupry, C.; Grant, D.M.; Pugmire, R.J. Carbon-13 magnetic resonance study of solvent stabilized tautomerism in pyrazoles. J. Org. Chem. 1977, 42, 659-661. [CrossRef]

45. Bergstrom, D.E.; Zhang, P.; Johnson, W.T. Design and synthesis of heterocyclic carboxamides as natural nucleic acid base mimics. Nucleosides Nucleotides 1996, 15, 59-68. [CrossRef]

46. Schneller, S.W. Carbocyclic nucleosides (Carbanucleosides) as new therapeutic leads. Curr. Top. Med. Chem. 2002, 2, 1087-1092. [CrossRef] 
47. Larsen, J.S.; Zahran, M.A.; Pedersen, E.B.; Nielsen, C. Synthesis of triazenopyrazole derivatives as potential inhibitors of HIV-1. Monatsh. Chem. 1999, 130, 1167-1173. [CrossRef]

48. Tominaga, Y.; Ushirogochi, A.; Matsuda, Y.; Koboyashi, G. Synthesis and reactions of 6-aryl- and 6-styryl-3-cyano-4-methylthio-2H-pyran-2-ones. Chem. Pharm. Bull. 1984, 32, 3384-3395. [CrossRef]

Sample Availability: Samples of the compounds are available from the corresponding author, V.S.P.

(C) 2019 by the authors. Licensee MDPI, Basel, Switzerland. This article is an open access article distributed under the terms and conditions of the Creative Commons Attribution (CC BY) license (http://creativecommons.org/licenses/by/4.0/). 